\title{
Parameter-dependent systems on the half-line and free convection boundary-layers in porous media
}

\author{
Gilles Evéquoz
}

\begin{abstract}
In this paper we use a recently elaborated abstract method, for parameter-dependent ODE systems over the interval $(0, \infty)$, to obtain existence results for the problem of self-similar solutions in boundarylayer free convection in porous media. Using a generalization of the method to exponentially decaying solutions, we are able to recover some known results, and to obtain a new branch of solutions in the case of the so-called backward boundary-layer. The arguments involve the derivation of suitable a priori estimates for the solutions of the problem.
\end{abstract}

Mathematics Subject Classification (2000). Primary 34A12;

Secondary 76R10.

Keywords. Global continuation, Ordinary differential equation,

A priori bounds, Boundary-layer, Free convection, Power-law similarity.

\section{Introduction}

The problem of the free convection along a vertical plate embedded in a porous medium has attracted considerable attention since the article by Cheng and Minkowicz [5] where the authors look at self-similar solutions. According to Magyari and Keller [14], the equations can be reduced to the ODE boundaryvalue problem (see Sect. 3 for more details)

$$
\left\{\begin{array}{l}
h^{\prime \prime \prime}(\tau)+s\left\{\frac{1}{2}(m+1) h(\tau) h^{\prime \prime}(\tau)-m\left(h^{\prime}(\tau)\right)^{2}\right\}=0, \quad \tau>0, \\
h(0)=h_{0}, \quad h^{\prime}(0)=1, \quad h^{\prime}(\infty)=0,
\end{array}\right.
$$

where $h_{0}, m \in \mathbb{R}$ and $s= \pm 1$ are given. The case $s=1$ is called "forward boundary layer" and corresponds to a heated plate, whereas it is called "backward boundary layer" when $s=-1$ and corresponds to a cooled plate. The 
parameter $h_{0}$ is linked to the amount of lateral suction/injection of mass flux through the permeable sheet.

In order to study the existence of solutions to (1.1), we shall use a recent method presented in [8-10], and which deals with parameter-dependent ODE systems over the half-line $(0, \infty)$. It is a continuation method in one of the parameters and relies upon a degree argument.

More precisely, let $J \subset \mathbb{R}$ and $U \subset \mathbb{R}^{k}$ be open subsets containing 0 , and consider a given decomposition $\mathbb{R}^{N}=X_{1} \oplus X_{2}$ with associated projection $P$ : $\mathbb{R}^{N} \rightarrow X_{1}$. We wish to study the behaviour of a connected component $\mathcal{C}$ of solutions $(\lambda, u, \xi)$ of the following boundary-value problem:

$$
\begin{aligned}
& \left\{\begin{array}{l}
u^{\prime}(t)+F(t, u(t), \xi, \lambda)=0, \quad t \in(0, \infty), \\
\operatorname{Pu}(0)=\varphi(\xi, \lambda),
\end{array}\right. \\
& \lim _{t \rightarrow \infty} u(t)=0
\end{aligned}
$$

where $F:[0, \infty) \times \mathbb{R}^{N} \times U \times J \longrightarrow \mathbb{R}^{N}$, and $\varphi: U \times J \longrightarrow X_{1}$ are given functions.

Problems of this kind, but with no parameter dependence have been considered by Rabier and Stuart $[21,22]$ in two recently published papers. The authors address the existence of solutions $u \in W^{1, p}\left((0, \infty), \mathbb{R}^{N}\right)$, with $1<p<\infty$, for systems of the form

$$
\left\{\begin{array}{l}
u^{\prime}(t)+F(t, u(t))=f(t), \quad \text { a.e. in }(0, \infty), \\
P u(0)=\zeta
\end{array}\right.
$$

where $f:[0, \infty) \rightarrow \mathbb{R}^{N}$ and $\zeta \in X_{1}$ are given.

Under the assumption that $F$ has a $t$-independent limit at $\infty$, Rabier and Stuart obtain existence results using a degree argument. Since the problem is set up over the unbounded interval $(0, \infty)$, the degree theory is not that of Leray and Schauder, but an extension of the latter to proper $C^{1}$ Fredholm mappings of index zero, constructed by Fitzpatrick et al. [11,19].

Problem (1.4) has also been studied by Morris [17] in his doctoral thesis. His work is based on the same degree theory, but the author looks for solutions $u$ in the space of $C^{1}$ functions vanishing at infinity. His choice is partly motivated by the fact that a priori bounds for the possible solutions are easier to obtain in this context than in the Sobolev setting. The results obtained by Morris do not involve the assumption that $F$ has a $t$-independent limit at infinity. The author replaces this assumption with a more general set of conditions on the so-called $\omega$-limit set of the function $F$ which contains all limits of $F(t, u)$ as $t \rightarrow \infty$, in an appropriate sense (see [17] for more details).

The originality in our approach to study (1.2) is to consider the set of parameters $\xi$ as part of the solution, whereas $\lambda$ is simply a continuation parameter. In principle, this allows us to treat situations where the dimension of $X_{1}$ (the number of prescribed initial values) is larger than was allowed in [21,22].

In the present paper, we discuss Problem (1.2) under the assumption that the function $F$ has a $t$-uniform asymptotic limit, as $t \rightarrow \infty$, for every value of the parameters $\xi$ and $\lambda$. We extend the continuation results of [9] in this case and, in addition, give conditions under which the existence of solutions 
of the form $\left(\lambda, e^{-\alpha t} v, \xi\right)$, where $v \in C_{0}^{1}\left([0, \infty), \mathbb{R}^{N}\right)$ and $\alpha>0$, is only subject to corresponding a priori bounds.

The paper is organized as follows. In Sect. 2, we recall briefly the functional setting which is used in studying Problem (1.2). For a detailed discussion we refer the reader to $[9,10]$. Next, we show how the conditions of Proposition 3.29 in [9] may be simplified in the case where $F$ has a limit as $t$ tends to $\infty$. In a second part, we discuss the existence of exponentially decaying solutions to $(1.2)$.

Section 3 is devoted to the study of (1.1). It starts with a brief description of the origin of the problem. With the help of a suitable change of variables, the system can be embedded into the functional setting discussed in Sect. 2. The verification of the hypotheses of the abstract continuation result is carried over through many steps. It requires a quite detailed and technical study of the behaviour of the possible solutions. Having established these conditions, we give an existence result for (1.1), which relies upon a priori bounds obtained via an integral formulation of the system (1.2). Some of the proofs and technical details have been postponed to Appendices A and B.

Summarizing our investigation of (1.1), we recover, in the forward boundary layer case $(s=1)$, only a part of the results already known in the literature. This situation has been almost completely solved by Brighi et al. [2-4]. We do not obtain any new result in this case.

For the backward boundary layer $(s=-1)$, our method gives a branch of solutions for every value of $m \neq-1$. In the literature, the situation is much more open. The problem for an impermeable plate $\left(h_{0}=0\right)$ has attracted much attention, but there are only few results about the permeable case. Let us mention the paper [13] by Liao which treats a physically different, but mathematically equivalent problem. Using his "homotopy analysis method", the author obtains two branches of solutions in the situation which corresponds for us to $m<-1$ and $h_{0}>0$. Magyari and Keller [14] consider the two special cases $m=-\frac{1}{3}$ and $m=1$ and find one, respectively two branches of solutions for the backward boundary layer. As far as we know, the branch obtained via our method has never been reported in the literature when $m>-1$ and $m \notin\left\{-\frac{1}{3}, 1\right\}$.

\section{Boundary-value systems with uniform asymptotic limit}

Let $\mathbb{R}^{N}=X_{1} \oplus X_{2}$ be a given decomposition with associated projection $P$ : $\mathbb{R}^{N} \rightarrow X_{1}$. Let $U \subset \mathbb{R}^{k}, J \subset \mathbb{R}$ be open subsets containing 0 , and assume that we are given two functions

$$
F:[0, \infty) \times \mathbb{R}^{N} \times U \times J \longrightarrow \mathbb{R}^{N} \quad \text { and } \varphi: U \times J \longrightarrow X_{1} .
$$

We start by recalling briefly the functional setting developed in [9] for the study of Problem (1.2).

Notation 2.1. Consider the following function spaces.

$$
\text { - } C_{b}^{1}\left(\mathbb{R}, \mathbb{R}^{N}\right):=\left\{u \in C^{1}\left(\mathbb{R}, \mathbb{R}^{N}\right): \sup _{t \in \mathbb{R}}\left(|u(t)|+\left|u^{\prime}(t)\right|\right)<\infty\right\} \text {. }
$$


- $C_{0}\left([0, \infty), \mathbb{R}^{N}\right)=\left\{u \in C\left([0, \infty), \mathbb{R}^{N}\right): \lim _{t \rightarrow \infty} u(t)=0\right\}$, endowed with the usual supremum norm $\|\cdot\|_{\infty}$.

- $C_{0}^{1}\left([0, \infty), \mathbb{R}^{N}\right)=\left\{u \in C^{1}\left([0, \infty), \mathbb{R}^{N}\right): \lim _{t \rightarrow \infty} u(t)=\lim _{t \rightarrow \infty} u^{\prime}(t)=0\right\}$, endowed with the norm $\|\cdot\|_{\infty, 1}$ defined by $\|u\|_{\infty, 1}=\|u\|_{\infty}+\left\|u^{\prime}\right\|_{\infty}$.

Definition 2.2. For every $(\lambda, u, \xi) \in J \times C_{0}^{1}\left([0, \infty), \mathbb{R}^{N}\right) \times U$, we let $\mathcal{F}(\lambda, u, \xi)(t)=\left(u^{\prime}(t)+F(t, u(t), \xi, \lambda), P u(0)-\varphi(\xi, \lambda)\right)$, for all $t \geq 0$.

Under the conditions $(\mathrm{C} 0)$ to $(\mathrm{C} 4)$ below, the operator

$$
\mathcal{F}: J \times C_{0}^{1}\left([0, \infty), \mathbb{R}^{N}\right) \times U \rightarrow C_{0}\left([0, \infty), \mathbb{R}^{N}\right) \times X_{1}
$$

is well-defined, continuous and differentiable with respect to $(u, \xi)$ (see Propositions 3.3 and 3.4 in [9]).

(C0) $\varphi \in C\left(U \times J, X_{1}\right), \varphi(0,0)=0$, and the partial derivative $D_{\xi} \varphi$ exists and is continuous on $U \times J$.

(C1) $F \in C\left([0, \infty) \times \mathbb{R}^{N} \times U \times J, \mathbb{R}^{N}\right)$, and $F(t, 0,0,0)=0, \forall t \geq 0$.

(C2) The partial derivatives $D_{u} F$ and $D_{\xi} F$ exist and are continuous on $[0, \infty) \times \mathbb{R}^{N} \times U \times J$.

(C3) $F, D_{u} F$, and $D_{\xi} F$ are bounded and uniformly continuous on $[0, \infty) \times$ $K \times W$, for all compact subsets $K \subset \mathbb{R}^{N}$ and $W \subset U \times J$.

(C4) For all $(\xi, \lambda) \in U \times J, \lim _{t \rightarrow \infty} F(t, 0, \xi, \lambda)=0$ and $\lim _{t \rightarrow \infty} D_{\xi} F(t, 0, \xi, \lambda)=0$.

Our approach is to study (1.2) via the functional equation $\mathcal{F}(\lambda, u, \xi)=0$. Assuming $\mathcal{F}(0,0,0)=0$, we wish to find out, whether a solution continues to exist for $\lambda \neq 0$. This will be achieved by considering the connected component of $\mathcal{F}^{-1}(0)$ which contains $(0,0,0)$.

In the present paper, we consider the special case where the following additional condition holds.

$\left(\mathrm{C}^{\infty}\right) \quad$ For every $(\xi, \lambda) \in U \times J$, there exists $F^{\infty}(\cdot, \xi, \lambda) \in C^{1}\left(\mathbb{R}^{N}, \mathbb{R}^{N}\right)$ such that $F^{\infty}(0, \xi, \lambda)=0$ and $\lim _{t \rightarrow \infty} D_{u} F(t, u, \xi, \lambda)=D_{u} F^{\infty}(u, \xi, \lambda)$, uniformly for $u$ in bounded subsets of $\mathbb{R}^{N}$.

\subsection{A continuation result}

We start by rewriting Proposition 3.29 of [9] under the assumption $\left(\mathrm{C}^{\infty}\right)$. The main point consists in reformulating the conditions (1) to (3) of that proposition for the problem with uniform limit. As is to be expected, the $\omega$-limit sets (see Definition 3.12 in [9]) of both $F$ and $D_{u} F$ reduce to a single element. Parts (a) and (b) of the next lemma follow by straightforward computations. The proof of Part (c) is similar to that of Theorem 2.3 in [21] and is left to the reader.

Lemma 2.3. Let the conditions (CO) to (C4) and $\left(C^{\infty}\right)$ be satisfied. For every $(\xi, \lambda) \in U \times J$, the following statements hold.

(a) $\omega(F(\cdot, \cdot, \xi, \lambda))=\left\{F^{\infty}(\cdot, \xi, \lambda)\right\}$.

(b) $\omega\left(D_{u} F(\cdot, 0, \xi, \lambda)\right)=\left\{D_{u} F^{\infty}(0, \xi, \lambda)\right\}$. 
(c) If $\sigma\left(D_{u} F^{\infty}(0, \xi, \lambda)\right) \cap i \mathbb{R}=\emptyset$, then $\operatorname{dim}\left(\operatorname{ker} D_{A}\right)=\operatorname{dim} X_{+}^{\infty}(\xi, \lambda)$, where $X_{+}^{\infty}(\xi, \lambda)$ denotes the positive generalized eigenspace ${ }^{1}$ of $D_{u} F^{\infty}(0, \xi, \lambda)$, and $D_{A} v=v^{\prime}+D_{u} F^{\infty}(0, \xi, \lambda) v$, for all $v \in C_{0}^{1}\left([0, \infty), \mathbb{R}^{N}\right)$.

Using Proposition 3.29 in [9] with the correction mentioned in [10], together with Lemma 2.3, we obtain the following.

Proposition 2.4. Assume that $F$ and $\varphi$ have the properties (CO) to (C4) and $\left(C^{\infty}\right)$ with $J \subset \mathbb{R}$ an open interval containing 0 and $U \subset \mathbb{R}^{k}$ an open and connected subset containing 0. Suppose that the following conditions are satisfied.

(1) For all $(\xi, \lambda) \in U \times J$, the set

$$
\left\{u \in C_{b}^{1}\left(\mathbb{R}, \mathbb{R}^{N}\right): u^{\prime}(t)+F^{\infty}(u(t), \xi, \lambda)=0, \forall t \in \mathbb{R}\right\}
$$

consists only of constant functions, and the set $\left\{u \in \mathbb{R}^{N}: F^{\infty}(u, \xi, \lambda)=\right.$ $0\}$ is totally disconnected. ${ }^{2}$

(2) For all $(\xi, \lambda) \in U \times J, \quad \sigma\left(D_{u} F^{\infty}(0, \xi, \lambda)\right) \cap i \mathbb{R}=\emptyset \quad$ and

$$
0 \leq k=\operatorname{dim} X_{1}-\operatorname{dim}\left(X_{+}^{\infty}(0,0)\right),
$$

where $X_{+}^{\infty}(0,0)$ is the positive generalized eigenspace of $D_{u} F^{\infty}$ $(0,0,0)$.

$$
\begin{gathered}
\left\{(v, \zeta) \in C_{0}^{1}\left([0, \infty), \mathbb{R}^{N}\right) \times \mathbb{R}^{k}: v^{\prime}+D_{(u, \xi)} F(\cdot, 0,0,0)(v, \zeta)=0\right. \text { and } \\
\left.P v(0)=D_{\xi} \varphi(0,0) \zeta\right\}=\{(0,0)\} .
\end{gathered}
$$

Let $\mathcal{O} \subset C_{0}^{1}\left([0, \infty), \mathbb{R}^{N}\right)$ be open and $0 \in \mathcal{O}$. Denote by $\mathcal{C}$ the maximal connected component of $\{(\lambda, u, \xi) \in J \times \mathcal{O} \times U: \mathcal{F}(\lambda, u, \xi)=0\}$ which contains $(0,0,0)$. If $\{(\lambda, u, \xi) \in \mathcal{C}: \lambda=0\}=\{(0,0,0)\}$, then the following holds.

(i) $\mathcal{C}_{+}$and $\mathcal{C}_{-}$are connected sets, where $\mathcal{C}_{+}=\{(\lambda, u, \xi) \in \mathcal{C}: \lambda \geq 0\}$ and $\mathcal{C}_{-}=\{(\lambda, u, \xi) \in \mathcal{C}: \lambda \leq 0\}$.

(ii) $\mathcal{C}_{+}$is unbounded, or at least one of $\overline{P_{\mathbb{R}}\left(\mathcal{C}_{+}\right)} \cap \partial J, P_{X}\left(\overline{\mathcal{C}_{+}}\right) \cap \partial \mathcal{O}$, and $\overline{P_{\mathbb{R}^{k}}\left(\mathcal{C}_{+}\right)} \cap \partial U$ is non-empty. ${ }^{3}$

(iii) $\mathcal{C}_{-}$is unbounded, or at least one of $\overline{P_{\mathbb{R}}\left(\mathcal{C}_{-}\right)} \cap \partial J, P_{X}\left(\overline{\mathcal{C}_{-}}\right) \cap \partial \mathcal{O}$, and $\overline{P_{\mathbb{R}^{k}}\left(\mathcal{C}_{-}\right)} \cap \partial U$ is non-empty.

The assumption (1) guarantees the properness property of the operator $\mathcal{F}$ given by Definition 2.2. As mentioned in [9], it is based upon a characterization of relatively compact subsets of $C_{0}\left(\mathbb{R}, \mathbb{R}^{N}\right)$ due to Rabier [20], and extended by Morris in [17] to the subsets of $C_{0}\left([0, \infty), \mathbb{R}^{N}\right)$. We also point out that in the assumption (2) we permit $k=0$, i.e. no parameter $\xi$. In Proposition 3.29 of [9], we required $k \geq 1$, since our approach actually takes its full extent when the parameter $\xi$ is needed. But in the latter, as well as in all results of [9], we can replace $k \geq 1$ by $k \geq 0$, since the arguments hold in this case also.

\footnotetext{
${ }^{1}$ Let $A \in \mathcal{L}\left(\mathbb{R}^{N}\right)$ satisfy $\sigma(A) \cap i \mathbb{R}=\emptyset$, and consider $\widetilde{X}_{+} \subset \mathbb{C}^{N}$ the direct sum of the generalized eigenspaces corresponding to eigenvalues of $A$ with positive real part. The positive generalized eigenspace of $A$ is the subspace $\left\{z \in \widetilde{X}_{+}: \operatorname{Im}(z)=0\right\} \subset \mathbb{R}^{N}$.

${ }^{2} \mathrm{~A}$ topological space $Z$ is called totally disconnected if, for every $x \in Z$, the intersection of all subsets of $Z$ which contain $x$ and are both open and closed is $\{x\}$. (See Engelking [7])

${ }^{3} P_{\mathbb{R}}, P_{X}$ and $P_{\mathbb{R}^{k}}$ denote the canonical projections from $\mathbb{R} \times C_{0}^{1}\left([0, \infty), \mathbb{R}^{N}\right) \times \mathbb{R}^{k}$ onto the first, second, and third factor, respectively. Moreover, $\overline{\mathcal{C}_{ \pm}}$denotes the closure of $\mathcal{C}_{ \pm}$in $\mathbb{R} \times C_{0}^{1}\left([0, \infty), \mathbb{R}^{N}\right) \times \mathbb{R}^{k}$.
} 


\subsection{Exponentially decaying solutions}

In this section we discuss the existence of solutions $(\lambda, u, \xi)$ of Problem (1.2) such that $u$ exhibits an exponential decay at infinity. More precisely, we require that there exist $\alpha>0$ and $v \in C_{0}^{1}\left([0, \infty), \mathbb{R}^{N}\right)$ satisfying

$$
u(t)=e^{-\alpha t} v(t), \text { for all } t \geq 0 .
$$

As we will see in Sect. 3, this framework allows us to deal with some situations in which the operator $\mathcal{F}$ is not Fredholm, that is, when condition (2) of Proposition 2.4 does not hold. Using the transformation (2.1), we will be able to recover the Fredholm property by an appropriate choice of the exponent $\alpha$.

If we rewrite $(1.2)$ using $(2.1)$, we find that $v \in C_{0}^{1}\left([0, \infty), \mathbb{R}^{N}\right)$ solves the following problem.

$$
\left\{\begin{array}{l}
v^{\prime}(t)+G(t, v(t), \xi, \lambda)=0, t \in(0, \infty), \\
P v(0)=\varphi(\xi, \lambda)
\end{array}\right.
$$

where, for all $(t, v, \xi, \lambda) \in[0, \infty) \times \mathbb{R}^{N} \times U \times J$,

$$
G(t, v, \xi, \lambda)=e^{\alpha t} F\left(t, e^{-\alpha t} v, \xi, \lambda\right)-\alpha v .
$$

As before, we define an operator, denoted by $\mathcal{G}$, whose zeros will correspond to the solutions of Problem (2.2).

Definition 2.5. For $(\lambda, v, \xi) \in J \times C_{0}^{1}\left([0, \infty), \mathbb{R}^{N}\right) \times U$, we let

$$
\mathcal{G}(\lambda, v, \xi)(t)=\left(v^{\prime}(t)+G(t, v(t), \xi, \lambda), P v(0)-\varphi(\xi, \lambda)\right) \text {, for all } t \geq 0 .
$$

In order to allow for a wider range of application of our method, we shall permit the exponent $\alpha$ to vary with the parameters $\xi$ and $\lambda$, according to the following condition.

$\left(\mathrm{G}^{\alpha}\right) \quad \alpha \in C(U \times J, \mathbb{R}), \alpha(\xi, \lambda)>0$ for all $(\xi, \lambda) \in U \times J$, and the partial derivative $D_{\xi} \alpha$ exists and is continuous on $U \times J$.

In order for $G$ and $\varphi$ to satisfy the conditions $(\mathrm{C} 0)$ to $(\mathrm{C} 4)$ we require the following sufficient conditions on $F$ and $\varphi$.

(G0) $\varphi \in C\left(U \times J, X_{1}\right), \varphi(0,0)=0$, and the partial derivative $D_{\xi} \varphi$ exists and is continuous on $U \times J$.

(G1) $F \in C\left([0, \infty) \times \mathbb{R}^{N} \times U \times J, \mathbb{R}^{N}\right)$, and $F(t, 0,0,0)=0$ for all $t \geq 0$.

(G2) The partial derivatives $D_{u} F$ and $D_{\xi} F$ exist and are continuous on $[0, \infty) \times \mathbb{R}^{N} \times U \times J$.

(G3) (i) The mappings $D_{u} F$ and $H$ are bounded and uniformly continuous on $[0, \infty) \times K \times W$, for all compact subsets $K \subset \mathbb{R}^{N}$ and $W \subset U \times J$, where

$$
\begin{aligned}
H(t, v, \xi, \lambda)= & t\left\{e^{\alpha(\xi, \lambda) t} F\left(t, e^{-\alpha(\xi, \lambda) t} v, \xi, \lambda\right)\right. \\
& \left.-D_{u} F\left(t, e^{-\alpha(\xi, \lambda) t} v, \xi, \lambda\right) v\right\} D_{\xi} \alpha(\xi, \lambda) \\
& +e^{\alpha(\xi, \lambda) t} D_{\xi} F\left(t, e^{-\alpha(\xi, \lambda) t} v, \xi, \lambda\right),
\end{aligned}
$$

for every $(t, v, \xi, \lambda) \in[0, \infty) \times \mathbb{R}^{N} \times U \times J$,

(ii) The mapping $(t, \xi, \lambda) \mapsto e^{\alpha(\xi, \lambda) t} F(t, 0, \xi, \lambda)$ is uniformly continuous on $[0, \infty) \times W$, for every compact subset $W \subset U \times J$. 
(G4) $\lim _{t \rightarrow \infty} e^{\alpha(\xi, \lambda) t} F(t, 0, \xi, \lambda)=0$, for all $(\xi, \lambda) \in U \times J$.

In addition, we assume that the following condition holds, so that $G$ will satisfy the assumption $\left(\mathrm{C}^{\infty}\right)$.

$\left(\mathrm{G}^{\infty}\right)$ For every $(\xi, \lambda) \in U \times J$, there exists $A^{\infty}(\xi, \lambda) \in \mathcal{L}\left(\mathbb{R}^{N}\right)$ such that

$$
\lim _{t \rightarrow \infty} D_{u} F(t, 0, \xi, \lambda)=A^{\infty}(\xi, \lambda) .
$$

Lemma 2.6. Suppose that $\alpha$ satisfies the condition $\left(G^{\alpha}\right)$ and that $F$ and $\varphi$ satisfy (GO) to (G4). Then $G$ and $\varphi$ satisfy the conditions (CO) to (C4). If in addition, $\left(G^{\infty}\right)$ holds, then $G$ also satisfies the condition $\left(C^{\infty}\right)$.

Proof. The properties (C0), (C1) and (C2) follow at once from (G0), $\left(\mathrm{G}^{\alpha}\right)$, (G1) and (G2).

For (C3), let $K \subset \mathbb{R}^{N}, W \subset U \times J$ be compact, and remark that $D_{u} G(t, v, \xi, \lambda)=D_{u} F\left(t, e^{-\alpha(\xi, \lambda) t} v, \xi, \lambda\right)-\alpha(\xi, \lambda) I$. By (G3), $D_{u} F$ is bounded and uniformly continuous on $[0, \infty) \times K^{\prime} \times W$, where

$$
K^{\prime}:=\overline{\left\{e^{-\alpha(\xi, \lambda) t} v:(t, v, \xi, \lambda) \in[0, \infty) \times K \times W\right\}}
$$

is a compact subset of $\mathbb{R}^{N}$. Hence, $D_{u} G$ is bounded and uniformly continuous on $[0, \infty) \times K \times W$.

Next, we observe that $D_{\xi} G(t, v, \xi, \lambda)=H(t, v, \xi, \lambda)-D_{\xi} \alpha(\xi, \lambda) v$, where $H$ is given by (2.4). The boundedness and uniform continuity of $H$, together with the continuity of $D_{\xi} \alpha$ imply that $D_{\xi} G$ is bounded and uniformly continuous on $[0, \infty) \times K \times W$.

It remains to prove the boundedness and uniform continuity of $G$. For this, we write

$$
G(t, v, \xi, \lambda)=G(t, 0, \xi, \lambda)+\int_{0}^{1} D_{u} G(t, s v, \xi, \lambda) v d s .
$$

Therefore, the boundedness and uniform continuity of $G(\cdot, 0, \cdot, \cdot)$, which follow from (G3)(ii) and (G4), and of $D_{u} G$, together with the compactness of $[0,1]$, give the desired conclusion.

Now we turn to (C4). We observe that (G4) gives $\lim _{t \rightarrow \infty} G(t, 0, \xi, \lambda)=0$, which is the first part of (C4). For the second part, we write for all $\eta \in \mathbb{R}^{k}$

$$
\lim _{t \rightarrow \infty} D_{\xi} G(t, 0, \xi, \lambda) \eta=\lim _{t \rightarrow \infty} \lim _{s \rightarrow 0} \frac{G(t, 0, \xi+s \eta, \lambda)-G(t, 0, \xi, \lambda)}{s} .
$$

Let $\delta>0$ be such that $\xi+s \eta \in U$, for all $|s| \leq \delta$. Since the continuous extension at $s=0$ of the map $(t, s) \mapsto \frac{1}{s}\{G(t, 0, \xi+s \eta, \lambda)-G(t, 0, \xi, \lambda)\}$ is uniformly continuous over $[0, \infty) \times[-\delta, \delta]$, by (G3)(ii), we may interchange the limits in (2.5), so that $\lim _{t \rightarrow \infty} D_{\xi} G(t, 0, \xi, \lambda)=0$.

Finally, we show that $\left(\mathrm{C}^{\infty}\right)$ holds with $G^{\infty}(v, \xi, \lambda):=A^{\infty}(\xi, \lambda) v-$ $\alpha(\xi, \lambda) v$, for all $(\lambda, v, \xi) \in J \times \mathbb{R}^{N} \times U$, provided $\left(\mathrm{G}^{\infty}\right)$ is satisfied.

Obviously, $G^{\infty}(\cdot, \xi, \lambda) \in C^{1}\left(\mathbb{R}^{N}, \mathbb{R}^{N}\right), G^{\infty}(0, \xi, \lambda)=0$, for all $(\xi, \lambda) \in$ $U \times J$ and $\left|D_{u} G(t, v, \xi, \lambda)-D_{u} G^{\infty}(v, \xi, \lambda)\right| \leq \mid D_{u} F\left(t, e^{-\alpha(\xi, \lambda) t} v, \xi, \lambda\right)-$ $D_{u} F(t, 0, \xi, \lambda)|+| D_{u} F(t, 0, \xi, \lambda)-A^{\infty}(\xi, \lambda) \mid$. Let $\bar{K} \subset \mathbb{R}^{N}$ be a bounded subset. Then

$$
\lim _{t \rightarrow \infty} e^{-\alpha(\xi, \lambda) t} v=0
$$


uniformly for $v \in K$, since $(\xi, \lambda)$ is fixed. Hence, the uniform continuity of $D_{u} F$ and the condition $\left(\mathrm{G}^{\infty}\right)$ conclude the proof.

We end this section by giving the analogue of Proposition 2.4 in the exponential setting.

Proposition 2.7. Let $\alpha$ satisfy the condition $\left(G^{\alpha}\right)$, and $F, \varphi$ have the properties (G0) to (G4) and $\left(G^{\infty}\right)$ with $J \subset \mathbb{R}$ an open interval containing 0 and $U \subset \mathbb{R}^{k}$ an open and connected subset containing 0 . Assume, in addition, that the following hypotheses are satisfied.

(1) For all $(\xi, \lambda) \in U \times J, \quad \sigma\left(A^{\infty}(\xi, \lambda)-\alpha(\xi, \lambda) I\right) \cap i \mathbb{R}=\emptyset$, and

$$
0 \leq k=\operatorname{dim} X_{1}-\operatorname{dim}\left(X_{+}^{\infty}(0,0)\right),
$$

where $X_{+}^{\infty}(0,0)$ is the positive generalized eigenspace of $\left(A^{\infty}(0,0)-\right.$ $\alpha(0,0) I)$.

(2) $\left\{(w, \zeta) \in C_{0}^{1}\left([0, \infty), \mathbb{R}^{N}\right) \times \mathbb{R}^{k}: w^{\prime}(t)+\left(D_{u} F(t, 0,0,0)-\alpha(0,0) I\right) w(t)\right.$ $+e^{\alpha(0,0) t} D_{\xi} F(t, 0,0,0) \zeta=0, t>0$ and $\left.P w(0)=D_{\xi} \varphi(0,0) \zeta\right\}=\{(0,0)\}$.

Let $\mathcal{V} \subset C_{0}^{1}\left([0, \infty), \mathbb{R}^{N}\right)$ be open and $0 \in \mathcal{V}$. Denote by $\mathcal{C}$ the maximal connected component of $\{(\lambda, v, \xi) \in J \times \mathcal{V} \times U: \mathcal{G}(\lambda, v, \xi)=0\}$ which contains $(0,0,0)$. If $\{(\lambda, v, \xi) \in \mathcal{C}: \lambda=0\}=\{(0,0,0)\}$, then the following holds.

(i) $\mathcal{C}_{+}$and $\mathcal{C}_{-}$are connected sets, where $\mathcal{C}_{+}=\{(\lambda, v, \xi) \in \mathcal{C}: \lambda \geq 0\}$ and $\mathcal{C}_{-}=\{(\lambda, v, \xi) \in \mathcal{C}: \lambda \leq 0\}$

(ii) $\mathcal{C}_{+}$is unbounded, or at least one of $\overline{P_{\mathbb{R}}\left(\mathcal{C}_{+}\right)} \cap \partial J, P_{X}\left(\overline{\mathcal{C}_{+}}\right) \cap \partial \mathcal{V}$, and $\overline{P_{\mathbb{R}^{k}}\left(\mathcal{C}_{+}\right)} \cap \partial U$ is non-empty.

(iii) $\mathcal{C}_{-}$is unbounded, or at least one of $\overline{P_{\mathbb{R}}\left(\mathcal{C}_{-}\right)} \cap \partial J, P_{X}\left(\overline{\mathcal{C}_{-}}\right) \cap \partial \mathcal{V}$, and $\overline{P_{\mathbb{R}^{k}}\left(\mathcal{C}_{-}\right)} \cap \partial U$ is non-empty.

Proof. By Lemma 2.6, $G$ satisfies the conditions $(\mathrm{C} 0)$ to $(\mathrm{C} 4)$ and $\left(\mathrm{C}^{\infty}\right)$ with $G^{\infty}(v, \xi, \lambda):=A^{\infty}(\xi, \lambda) v-\alpha(\xi, \lambda) v$.

Moreover, since $\sigma\left(A^{\infty}(\xi, \lambda)-\alpha(\xi, \lambda) I\right) \cap i \mathbb{R}=\emptyset$ holds, we find that $\left\{v \in C_{b}^{1}\left(\mathbb{R}, \mathbb{R}^{N}\right): v^{\prime}+\left(A^{\infty}(\xi, \lambda)-\alpha(\xi, \lambda) I\right) v=0\right\}=\{0\}$ (see for example the criterion (12.14) on page 183 in [1]), and $\left\{v \in \mathbb{R}^{N}:\left(A^{\infty}(\xi, \lambda)-\alpha(\xi, \lambda) I\right) v\right.$ $=0\}=\{0\}$.

Replacing $F$ and $F^{\infty}$ by $G$ and $G^{\infty}$, respectively, in the conditions (2) and (3) of Proposition 2.4 gives exactly the assumptions (1) and (2) of the present proposition. Therefore, the conclusion follows from the former proposition applied to the operator $\mathcal{G}$.

\section{Solutions of a boundary-layer problem}

As an application of the general results obtained in the previous section, we now discuss the following problem of boundary-layer free convection in a porous medium. (For more details, we refer the reader to $[5,14,18]$.)

Consider a semi-infinite vertical plate and assume that the whole region to the right of the plate consists of a porous medium saturated by some convective fluid with ambient temperature $\theta_{\infty}$. The coordinate system is chosen according to Fig. 1. The origin is set on the finite edge of the plate, and the 


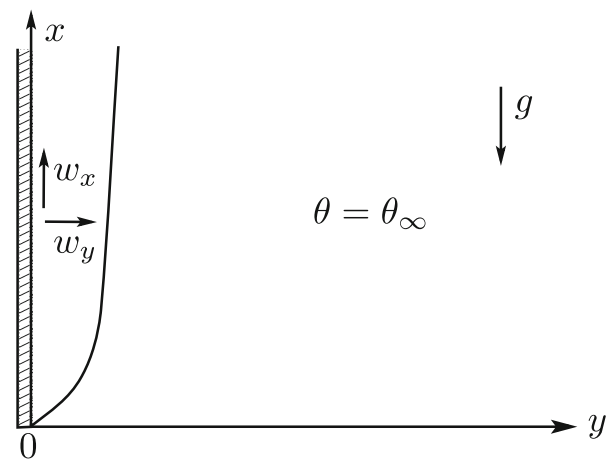

FiguRE 1. Flow domain of a free convection boundary-layer

$x$-axis is directed vertically upwards, in the opposite direction to the gravity field $g$. The temperature distribution along the wall is assumed to follow a power law, i.e.

$$
\theta(x, 0)=\theta_{\infty}+s \theta_{0} x^{m},
$$

with $\theta_{0}>0, m \in \mathbb{R}$ and $s= \pm 1$. We speak of a 'heated' (resp. 'cooled') plate when $s=1$, (resp. $s=-1)$ since $\theta(x, 0)>\theta_{\infty}\left(\operatorname{resp} . \theta(x, 0)<\theta_{\infty}\right)$ for $x>0$. According to Magyari and Keller [14], the dimensionless stream function $h$ satisfies the boundary-value problem

$$
\begin{aligned}
& h^{\prime \prime \prime}(\tau)+s\left\{\frac{1}{2}(m+1) h(\tau) h^{\prime \prime}(\tau)-m\left(h^{\prime}(\tau)\right)^{2}\right\}=0, \quad \tau>0, \\
& h(0)=h_{0}, \quad h^{\prime}(0)=1, \quad h^{\prime}(\infty)=0,
\end{aligned}
$$

where $h_{0} \in \mathbb{R}$.

The relation with the physical variables is given by the following. Denote by $\theta$ the local temperature of the fluid inside the boundary-layer, and by $w_{x}$, $w_{y}$ the velocity components along the $x$ and $y$ axes. Let $\psi$ be the stream function, so that $w_{x}=\frac{\partial \psi}{\partial y}$ and $w_{y}=-\frac{\partial \psi}{\partial x}$, then

$$
\psi(x, y)=s \nu \sqrt{R a} x^{\frac{m+1}{2}} h(\tau), \quad \tau=\sqrt{R a} x^{\frac{m-1}{2}} y,
$$

$\nu$ being the thermal diffusivity, and $R a$ the Rayleigh number in a porous medium. The temperature and velocity components are then given by

$$
\begin{aligned}
\theta(x, y) & =\theta_{\infty}+s \theta_{0} x^{m} h^{\prime}(\tau), \\
w_{x}(x, y) & =s \nu \operatorname{Ra} x^{m} h^{\prime}(\tau), \\
w_{y}(x, y) & =-s \frac{\nu \sqrt{R a}}{2} x^{\frac{m-1}{2}}\left[(m+1) h(\tau)+(m-1) \tau h^{\prime}(\tau)\right] .
\end{aligned}
$$

Furthermore, $h_{0}$ satisfies the relation

$$
w_{y}(x, 0)=-s h_{0}(m+1) \frac{\nu \sqrt{R a}}{2} x^{\frac{m-1}{2}} .
$$

When $w_{y}(x, 0)=0$ the plate is impermeable, whereas lateral suction (resp. injection) of fluid is applied to the plate when $w_{y}(x, 0)<0\left(\right.$ resp. $w_{y}(x, 0)>$ 
$0)$. The parameter $h_{0}$ can therefore be understood as the dimensionless suction/injection velocity (see [14]).

\subsection{Statement of the problem in the functional setting}

As mentioned in the beginning of this section, we would like to use the method discussed in Sect. 2 to find solutions of the system (3.1) and (3.2). For that purpose, we need to have a special solution from which we may start the continuation procedure. With this respect, we remark that the obvious choice $h \equiv h_{0}$ is not admissible, since $(3.2)$ requires $h^{\prime}(0)=1$.

In order to allow for such a solution, we restrict our study of (3.1) and (3.2) to the suction case (see (3.3)):

$$
s h_{0}(m+1)>0,
$$

and make the following change of variables.

$$
t=s h_{0}(m+1) \tau \quad \text { and } \quad f(t)=\frac{1}{h_{0}} h(\tau) .
$$

Hence (3.1) and (3.2) become

$$
\begin{aligned}
& f^{\prime \prime \prime}(t)+\frac{1}{2} f(t) f^{\prime \prime}(t)-\frac{m}{m+1}\left(f^{\prime}(t)\right)^{2}=0, \quad t>0, \\
& f(0)=1, \quad f^{\prime}(0)=\lambda, \quad f^{\prime}(\infty)=0,
\end{aligned}
$$

where

$$
\lambda=\frac{1}{s h_{0}^{2}(m+1)} .
$$

Now $f \equiv 1$ is a solution of (3.6) and (3.7) for $\lambda=0$. Notice that the condition (3.4) excludes the case $m=-1$. Solutions of (3.6) and (3.7) with $\lambda(m+1) \gtrless 0$ will correspond to solutions of (3.1) and (3.2) with $s= \pm 1$ and $h_{0}(m+1) \gtrless 0$. Moreover, $h_{0}$ and $\lambda$ will have the same sign..

The next step towards the formulation in the functional setting is the choice of the parameter $\xi$. Recall that the method in Sect. 2 deals with functions vanishing at infinity. In view of the condition $f^{\prime}(\infty)=0$, we may either look for solutions satisfying $\lim _{t \rightarrow \infty} f(t)=0$, which would be quite restrictive, or more generally, seek solutions which have a finite limit as $t \rightarrow \infty$. For this reason, we set

$$
\xi:=\lim _{t \rightarrow \infty} f(t)-1
$$

Remark 3.1. Although the parameter $\xi$ does not come out as a natural parameter, the introduction of the value of $f$ at infinity is not uncommon in the context of boundary-layer problems. For example, Von Kármán and Lin [12], followed by many other authors, used it in the context of a viscous flow above an infinite rotating disc. Concerning the present problem, the same idea has been used by Liao [13] when applying his homotopy analysis method, but it does not seem to be so widely used. Indeed, most of the papers treating this problem introduce the value of $f^{\prime \prime}(0)$ as a parameter. This choice, however, does not fit well in our framework, since it gives us an initial-value problem, 
and it does not seem so obvious, then, how to control the behaviour of $f$ at infinity.

Setting $X_{1}=\operatorname{span}\left\{e_{1}, e_{2}\right\} \subset \mathbb{R}^{3}$, and

$$
u(t)=\left(f(t)-1-\xi, f^{\prime}(t), f^{\prime \prime}(t)\right), \quad \text { for all } t \geq 0,
$$

we can rewrite (3.6) as the first-order system (1.2), with

$$
F(t, u, \xi, \lambda)=A(\xi) u+N(u)
$$

and

$$
\varphi(\xi, \lambda)=(-\xi, \lambda, 0)
$$

where

$$
\begin{aligned}
A(\xi) & =\left(\begin{array}{ccc}
0 & -1 & 0 \\
0 & 0 & -1 \\
0 & 0 & \frac{1}{2}(1+\xi)
\end{array}\right), \\
N(u) & =\left(0,0, \frac{1}{2} u_{1} u_{3}-\frac{m}{m+1} u_{2}^{2}\right),
\end{aligned}
$$

and $(t, u, \xi, \lambda) \in[0, \infty) \times \mathbb{R}^{3} \times U \times \mathbb{R}$. The set $U \subset \mathbb{R}$ will be chosen subsequently as an open subinterval of $(-1, \infty)$ containing zero and depending upon $m$.

The first idea would be to use Proposition 2.4 to obtain information about the branch of solutions containing the trivial solution. Unfortunately, condition (2) of this proposition is not going to hold in our case. Indeed, since the map $F$ does not depend upon $t$, any choice for $F^{\infty}(u, \xi, \lambda)$ will give us $D_{u} F^{\infty}(0, \xi, \lambda)=D_{u} F(0,0, \xi, \lambda)=A(\xi)+N^{\prime}(0)=A(\xi)$. But we see immediately that $0 \in \sigma(A(\xi))$.

To overcome this problem, we try looking for exponentially decaying solutions, and therefore use Proposition 2.7 instead of Proposition 2.4. According to Definition 2.5 we are now looking for solutions $(\lambda, v, \xi)$ of the system $(2.2)$, with

$$
G(t, v, \xi, \lambda)=(A(\xi)-\alpha(\xi, \lambda) I) v+e^{-\alpha(\xi, \lambda) t} N(v),
$$

for $(t, v, \xi, \lambda) \in[0, \infty) \times \mathbb{R}^{3} \times U \times \mathbb{R}$, and where $\alpha(\xi, \lambda)$ is to be chosen.

Lemma 3.2. Let $m \in \mathbb{R} \backslash\{-1\}$ be fixed, $J=\mathbb{R}$, and $U$ be any open subinterval of $(-1, \infty)$ containing zero. For every function $\alpha$ satisfying $\left(G^{\alpha}\right)$ and the condition

$$
0<\alpha(\xi, \lambda)<\frac{1}{2}(1+\xi), \quad \text { for all }(\xi, \lambda) \in U \times J,
$$

the functions $F$ and $\varphi$ defined by (3.10) and (3.11) satisfy (G0) to (G4) and $\left(G^{\infty}\right)$. Furthermore, the conditions (1) and (2) of Proposition 2.7 are fulfilled.

Proof. The conditions (G0), (G1) and (G2) are obviously satisfied. Concerning (G3)(i), we let $K \subset \mathbb{R}^{3}$ and $W \subset U \times J$ be compact. Since $D_{u} F(t, u, \xi, \lambda)=$ 
$A(\xi)+N^{\prime}(u)$, for all $(t, u, \xi, \lambda)$, does not depend upon $t$, it is bounded and uniformly continuous on $[0, \infty) \times K \times W$. Next, we have

$$
H(t, v, \xi, \lambda)=-\alpha(\xi, \lambda) t e^{-\alpha(\xi, \lambda) t} N(v)+\frac{d}{d \xi} A(\xi) v .
$$

Since $\lim _{t \rightarrow \infty} t e^{-\alpha(\xi, \lambda) t}=0$, the continuity of $\alpha$ and $N$ imply that $H$ is bounded and uniformly continuous on $[0, \infty) \times K \times W$. Moreover, $e^{\alpha(\xi, \lambda) t} F(t, 0, \xi, \lambda)=0$ for all $(t, \xi, \lambda) \in[0, \infty) \times U \times J$, from which we see that (G3)(ii) and (G4) hold. Finally, $\left(\mathrm{G}^{\infty}\right)$ holds with $A^{\infty}=A$. Indeed, $\lim _{t \rightarrow \infty} D_{u} F(t, 0, \xi, \lambda)=A(\xi)+N^{\prime}(0)=A(\xi)$, for every $(\xi, \lambda) \in U \times J$.

Let us check the conditions of Proposition 2.7.

(1) For every $\xi \in U,(A(\xi)-\alpha(\xi, \lambda) I)$ has always one negative (double) eigenvalue, $\lambda_{1}=-\alpha(\xi, \lambda)$, and one positive (simple) eigenvalue, $\lambda_{2}=$ $\frac{1}{2}(1+\xi)-\alpha(\xi, \lambda)$. Therefore, $\operatorname{dim} X_{1}-\operatorname{dim} X_{+}^{\infty}(\xi, \lambda)=1=k$, for every $(\xi, \lambda) \in U \times J$.

(2) We have to show that $(w, \zeta)=(0,0)$ is the only solution in $C_{0}^{1}\left([0, \infty), \mathbb{R}^{3}\right)$ $\times \mathbb{R}$ of the system

$$
\begin{cases}w^{\prime}(t)+(A(0)-\alpha(0,0) I) w(t)=0, & t>0 \\ w_{1}(0)=-\zeta, & w_{2}(0)=0 .\end{cases}
$$

Solving the initial value-problem, with $w_{3}(0)$ unknown, we obtain

$$
\left\{\begin{array}{l}
w_{1}(t)=-\zeta e^{\alpha(0,0) t}+4 w_{3}(0) e^{\alpha(0,0) t}\left(\frac{1}{2} t-1+e^{-\frac{1}{2} t}\right) \\
w_{2}(t)=2 w_{3}(0) e^{\alpha(0,0) t}\left(1-e^{-\frac{1}{2} t}\right) \\
w_{3}(t)=w_{3}(0) e^{\left(\alpha(0,0)-\frac{1}{2}\right) t}
\end{array}\right.
$$

Since $w \in C_{0}^{1}\left([0, \infty), \mathbb{R}^{3}\right)$, we must have $\lim _{t \rightarrow \infty} w_{1}(t)=\lim _{t \rightarrow \infty} w_{2}(t)=0$, and therefore $w_{3}(0)=0$, and $\zeta=0$, since $\alpha(0,0)>0$. Consequently, $w_{1}(t)=w_{2}(t)=w_{3}(t)=\zeta=0$, for all $t \geq 0$.

Let $\mathcal{G}$ be defined by $(2.5)$, with $G$ given by (3.14), and denote by $\mathcal{C}$ the connected component of $\left\{(\lambda, v, \xi) \in J \times C_{0}^{1}\left([0, \infty), \mathbb{R}^{3}\right) \times U: \mathcal{G}(\lambda, v, \xi)=0\right\}$ which contains $(0,0,0)$. According to Lemma 3.2 , the choice $J=\mathbb{R}$, and $U=(-1, \infty)$ seems to be the best one. But Proposition 2.7 requires that $(0,0,0)$ be the only solution $(\lambda, v, \xi)$ in $\mathcal{C}$ for which $\lambda=0$. In order to fulfill this condition, we need more information about the solutions of (3.6) and (3.7), and this is the purpose of the next section. As we will see, the choice of the set $U$ will depend upon the value of the parameter $m$, and will in most cases be a strict subinterval of $(-1, \infty)$.

\subsection{Properties of the solutions of (3.6) and (3.7)}

In this section, we wish to derive some properties of the solutions of (3.6) and (3.7), which will allow us to choose the set $U$ in a suitable way, and to obtain a priori bounds on the solutions of (2.2). This section is rather technical and, for this reason, some of the proofs are postponed to Appendix A. 
Remark 3.3. Let $(\lambda, v, \xi)$ be a solution of (2.2). The function $f$ defined by

$$
f(t)=e^{-\alpha(\xi, \lambda) t} v_{1}(t)+(1+\xi), \quad \text { for all } t \geq 0
$$

is a solution of (3.6) and (3.7). Moreover, for all $t \geq 0$,

$$
f^{\prime}(t)=e^{-\alpha(\xi, \lambda) t} v_{2}(t), \quad f^{\prime \prime}(t)=e^{-\alpha(\xi, \lambda) t} v_{3}(t), \quad \text { and } \quad \lim _{t \rightarrow \infty} f(t)=1+\xi .
$$

The next result discusses the behaviour of the solutions of (3.6), which satisfy the boundary condition $f^{\prime}(\infty)=0$. In particular, we show that the solutions are ultimately monotone, with at most one concavity change. Moreover, for some values of $m$ (see (3) below) all solutions have a nonnegative and finite limit at infinity. The last part of the lemma provides a criterion for the existence of the concavity change, and is a crucial ingredient of the forthcoming results.

Lemma 3.4. Let $m \in \mathbb{R} \backslash\{-1\}$ and consider a solution $f \in C^{3}([0, \infty), \mathbb{R})$ of (3.6) which satisfies $\lim _{t \rightarrow \infty} f^{\prime}(t)=0$ and $f^{\prime} \not \equiv 0$. The following statements hold.

(1) $f^{\prime}$ has at most one critical point in $[0, \infty)$. Moreover, if $t^{*} \geq 0$ satisfies $f^{\prime \prime}\left(t^{*}\right)=0$, then $\frac{m}{(m+1)}\left(t-t^{*}\right) f^{\prime \prime}(t)>0$ for all $t \neq t^{*}$.

(2) $f^{\prime \prime}$ is ultimately monotone, and $\lim _{t \rightarrow \infty} f^{\prime \prime}(t)=0$. Moreover, if $f(t) \geq 0$ for all $t \geq 0$, then $f^{\prime \prime}$ has at most one critical point.

(3) $f$ has at most one critical point in $[0, \infty)$. Furthermore, if $f(0)>0$ and $m \in(-\infty,-1) \cup[0, \infty)$, then $f(t)>0$ for all $t \geq 0$, and

$$
0 \leq \lim _{t \rightarrow \infty} f(t)<+\infty
$$

(4) If $\lim _{t \rightarrow \infty} f(t)$ is finite then, for all $t \geq 0$,

$$
\begin{aligned}
& -f^{\prime \prime}(t)-\frac{1}{2} f(t) f^{\prime}(t)=\frac{3 m+1}{2(m+1)} \int_{t}^{+\infty}\left(f^{\prime}(s)\right)^{2} d s . \\
& -f(t) f^{\prime \prime}(t)+\frac{1}{2}\left(f^{\prime}(t)\right)^{2}-\frac{1}{2}(f(t))^{2} f^{\prime}(t) \\
& =\frac{2 m+1}{(m+1)} \int_{t}^{+\infty} f(s)\left(f^{\prime}(s)\right)^{2} d s .
\end{aligned}
$$

(5) Suppose $f(0)>0, \lim _{t \rightarrow \infty} f(t)=:(1+\xi)$ is finite, and there exists $t^{*} \geq 0$ for which $f^{\prime \prime}\left(t^{*}\right)=0$. Then $m \notin\left[-\frac{1}{3}, \frac{1}{3}\right]$ and

$$
\frac{2 m}{(m+1)} f\left(t^{*}\right)>\frac{(3 m+1)}{(m+1)}(1+\xi) \text {. }
$$

The proof is given in Appendix A.

Lemma 3.5. Let $m \in \mathbb{R} \backslash\{-1\}$ and consider a solution $f \in C^{3}([0, \infty), \mathbb{R})$ of (3.6) and (3.7) such that $\lim _{t \rightarrow \infty} f(t)=:(1+\xi)$ is finite.

(a) If $m \in(-\infty,-1) \cup\left[-\frac{1}{3}, \infty\right)$, then $(1+\xi)^{2} \leq 1+4 \lambda$.

(b) If $m \in\left[-\frac{1}{2},-\frac{1}{3}\right)$ and $f(t) \geq 0$ for all $t \geq 0$, then $(1+\xi)^{3} \leq 1+6 \lambda-$ $\frac{9(m+1)}{(3 m+1)} \lambda^{2}$. 
(c) Suppose $\xi>-1$ and $f^{\prime \prime}(t) \neq 0$ for all $t \geq 0$. Then for every $t \geq 0$, $|f(t)-(1+\xi)| \leq|\xi|, \quad\left|f^{\prime}(t)\right| \leq|\lambda|, \quad$ and $\quad\left|f^{\prime \prime}(t)\right| \leq \frac{1}{2}|\lambda|+\frac{(4|m|+1)}{2|m+1|} \lambda^{2}$.

(d) Suppose $m \in\left[-\frac{1}{2},-\frac{1}{3}\right), \lambda>0$, and there exists some $t^{*} \geq 0$ such that $f^{\prime \prime}\left(t^{*}\right)=0$. Then for every $t \geq 0$,

$$
|f(t)-(1+\xi)| \leq \xi, \quad\left|f^{\prime}(t)\right| \leq \frac{\xi}{4}(2+\xi), \text { and }\left|f^{\prime \prime}(t)\right| \leq \frac{\xi^{2}}{8}(2+\xi)^{2} .
$$

The proof is given in Appendix A.

We conclude this study of the solutions of (3.6) and (3.7) with a nonexistence result, already obtained by Brighi for $m \leq-1$ (see Theorem 3.1 in [2]). It is an immediate consequence of Lemma 3.5(a) and Lemma 3.4(3).

Corollary 3.6. (non-existence) If $m \in(-\infty,-1) \cup[0, \infty)$, the system (3.6) and (3.7) has no solution $f \in C^{3}\left([0, \infty), \mathbb{R}^{3}\right)$ with $\lambda<-\frac{1}{4}$. Moreover, if $m \in\left[-\frac{1}{3}, 0\right)$, no solution of class $C^{3}$ with $\lambda<-\frac{1}{4}$ is bounded over $[0, \infty)$.

We now go back to the system (2.2), and check the remaining condition of Proposition 2.7. In view of the preceding Lemma, we are in position to choose the set $U$ in a suitable way for the trivial solution to be the only solution for which $\lambda=0$. Indeed, as we will see below, this can be achieved by choosing $U$ so that (3.19) does not hold. (For the notation $\mathcal{C}_{ \pm}$below, we refer to Proposition 2.7, with $\mathcal{V}=C_{0}^{1}\left([0, \infty), \mathbb{R}^{3}\right)$.)

Proposition 3.7. Let $m \in \mathbb{R} \backslash\{-1\}, J=\mathbb{R}$,

$$
U= \begin{cases}\left(-\frac{(m+1)}{(3 m+1)}, \infty\right), & \text { if } m \in(-\infty,-1) \cup\left(\frac{1}{3}, \infty\right), \\ \left(-1,-\frac{(m+1)}{(3 m+1)}\right), & \text { if } m \in\left(-1,-\frac{1}{2}\right), \\ (-1, \infty), & \text { if } m \in\left[-\frac{1}{2}, \frac{1}{3}\right],\end{cases}
$$

and consider a function $\alpha=\alpha(\xi, \lambda)$ satisfying $\left(G^{\alpha}\right)$ and (3.15). The following statements hold.

(1) $\mathcal{C}_{+} \cap \mathcal{C}_{-}=\{(0,0,0)\}$.

(2) $\operatorname{Let}(\lambda, v, \xi) \in \mathcal{C}_{+} \backslash\{(0,0,0)\}$.

- If $m \in\left(-\infty,-\frac{1}{2}\right) \cup\left[-\frac{1}{3}, \infty\right)$, then $v_{3}(t)<0$, for all $t \geq 0$.

- If $m \in\left[-\frac{1}{2},-\frac{1}{3}\right)$, either $v_{3}(t)<0$ for all $t \geq 0$, or there exists a unique $t^{*} \in\left[0,-\frac{(m+1)}{(3 m+1)}\right]$ with $v_{3}\left(t^{*}\right)=0$ and $\left(t-t^{*}\right) v_{3}(t)<0$ for all $t \neq t^{*}$.

(3) $\operatorname{Every}(\lambda, v, \xi) \in \mathcal{C}_{-} \backslash\{(0,0,0)\}$ satisfies $v_{3}(t)>0$, for all $t \geq 0$.

Proof. (1) Let $(\lambda, v, \xi) \in \mathcal{C}_{+} \cap \mathcal{C}_{-}$, so that $\lambda=0$, and suppose by contradiction that $v \not \equiv 0$. Using Remark 3.3, we define the corresponding solution $f$ of (3.6) and (3.7), via (3.16). Since $\lim _{t \rightarrow \infty} f^{\prime}(t)=0$, there must exist some $t^{*}>0$ for which $f^{\prime \prime}\left(t^{*}\right)=0$, and $t^{*}$ is unique, by Lemma 3.4(1).

Also $\lim _{t \rightarrow \infty} f(t)=1+\xi$ is finite and $f(0)=1$. Thus, Lemma 3.4(5) gives the desired contradiction in the case $m \in\left[-\frac{1}{3}, \frac{1}{3}\right]$. 
Next, we look at the case where $m \in\left[-\frac{1}{2},-\frac{1}{3}\right]$. By Lemma 3.4(1), $\left(t-t^{*}\right) f^{\prime \prime}(t)<0$ for all $t \neq t^{*}$. This implies that $f^{\prime}(t)>0$ and $f(t)>1$ for all $t>0$. Evaluating (3.18) at $t=0$, we obtain $f^{\prime \prime}(0) \leq 0$ which contradicts the previous statement, since $t^{*}>0$. Hence, the result holds in this case also.

Finally, if $m \in\left(-\infty,-\frac{1}{2}\right) \cup\left(\frac{1}{3}, \infty\right)$, it follows from our choice of $U$ that $\frac{(3 m+1)}{(m+1)}(1+\xi) \geq \frac{2 m}{(m+1)}$. Since the uniqueness of $t^{*}$ implies that $f^{\prime}$ cannot vanish in $(0, \infty)$, we must have $\frac{m}{m+1} f^{\prime}(t)<0$ for all $t>0$, by (3.6). Therefore, we obtain $\frac{(3 m+1)}{(m+1)}(1+\xi) \geq \frac{2 m}{(m+1)} f(0)>\frac{2 m}{(m+1)} f\left(t^{*}\right)$, which contradicts Part (5) of Lemma 3.4, and proves the result.

(2) Take $(\lambda, v, \xi) \in \mathcal{C}_{+} \backslash\{(0,0,0)\}$, and define $f$ by (3.16). Then $f$ is a solution of (3.6), (3.7), and $\lambda>0$, from (1) above.

First remark that the result is already true for $m \in\left[-\frac{1}{3}, \frac{1}{3}\right]$. Indeed, from Lemma $3.4(5)$, we know that $f^{\prime \prime}$ never vanishes in $[0, \infty)$, so that $f^{\prime \prime}(t)<0$ for all $t \geq 0$. Using Remark 3.3, this means that $v_{3}(t)<0$, for all $t \geq 0$.

If $m \in\left[-\frac{1}{2},-\frac{1}{3}\right)$, we obtain from Lemma 3.4(1) that either $f^{\prime \prime}(t)<$ 0 for all $t \geq 0$ ( since $\lambda>0$ ), or there is a unique $t^{*} \geq 0$ for which $f^{\prime \prime}\left(t^{*}\right)=0$, in which case $\left(t-t^{*}\right) f^{\prime \prime}(t)<0$ for all $t \neq t^{*}$. In the latter case, $0<\lambda \leq f^{\prime}(t)$ for all $0 \leq t \leq t^{*}$, and from (3.17) we obtain

$-f^{\prime \prime}(0)-\frac{1}{2} \lambda+\frac{1}{2} f\left(t^{*}\right) f^{\prime}\left(t^{*}\right)=\frac{(3 m+1)}{2(m+1)} \int_{0}^{t^{*}}\left(f^{\prime}(s)\right)^{2} d s \leq \frac{(3 m+1)}{2(m+1)} \lambda^{2} t^{*}$.

But $f\left(t^{*}\right) f^{\prime}\left(t^{*}\right)>0$, and (3.18) at $t=0$ gives $f^{\prime \prime}(0) \leq \frac{1}{2}\left(\lambda^{2}-\lambda\right)$ (hence, $t^{*}$ exists only if $\lambda \geq 1$ ). Thus, in view of the inequality above, we find $-\frac{1}{2} \lambda^{2} \leq \frac{(3 m+1)}{2(m+1)} \lambda^{2} t^{*}$, which gives the desired bound for $t^{*}$.

Now we suppose that $m \in\left(-1,-\frac{1}{2}\right)$. In this case, it follows from Lemma 3.4(1) that $f^{\prime}(t)>0$ for all $t \geq 0$. Thus, if $f^{\prime \prime}$ vanishes at some $t^{*} \geq 0$, then $1=f(0) \leq f\left(t^{*}\right)<(1+\xi)$, and from Lemma 3.4(5), $1+\xi>\frac{2 m}{3 m+1}$. But this is impossible, from our choice of $U$. Therefore, $f^{\prime \prime}$ does not vanish in $[0, \infty)$ and, by Remark 3.3, $v_{3}(t)<0$, for all $t \geq 0$.

The last case to consider occurs when $m \in(-\infty,-1) \cup\left(\frac{1}{3}, \infty\right)$. We will use the connectedness of $\mathcal{C}_{+}$, which follows from Proposition 2.7 (i) and Part (1) above, to prove the desired result. For this purpose, we set

$$
\Sigma=\left\{(\lambda, v, \xi) \in \mathcal{C}_{+}: v_{3}(t)<0, \text { for all } t \geq 0\right\} \cup\{(0,0,0)\} .
$$

Let $(\lambda, v, \xi)$ belong to the closure of $\Sigma$ with respect to $\mathcal{C}_{+}$. Then $\lambda \geq 0$, $v_{3}(t) \leq 0$ for all $t \geq 0$. Defining the associated function $f$ by (3.16), this means that $f^{\prime \prime}(t) \leq 0$ for all $t \geq 0$. But Lemma 3.4(1) implies that if $f^{\prime} \not \equiv 0$ and $f^{\prime \prime}$ vanishes at some point, then it has to change sign. Therefore, either $f^{\prime} \equiv 0$, i.e. $(\lambda, v, \xi)=(0,0,0)$, or $f^{\prime \prime}(t)<0$, i.e. $v_{3}(t)<0$, for all $t \geq 0$, in which case, $\lambda>0$ and $(\lambda, v, \xi) \in \Sigma$. Thus, $\Sigma$ is closed in $\mathcal{C}_{+}$.

It remains to show that $\Sigma$ is open in $\mathcal{C}_{+}$. For this we consider $(\lambda, v, \xi) \in \Sigma$ and set $r=\frac{(m+1)}{(3 m+1)}(1+\xi)>0$. Let $(\mu, w, \eta) \in \mathcal{C}_{+}$be such that $\|(\mu, w, \eta)-(\lambda, v, \xi)\|<r$. Since $\mu \geq 0$, Part (1) above gives that either 
$(\mu, w, \eta)=(0,0,0)$, in which case the property is established, or $\mu>0$. In the second case we define the functions $f$ and $g$ associated to $(\lambda, v, \xi)$ and $(\mu, w, \eta)$, respectively, via (3.16). Since $\lambda>0$ and $f^{\prime \prime}(t)<0$ for all $t \geq 0$, we know that $1 \leq f(t)<1+\xi$, for all $t \geq 0$. Moreover, $\eta>\xi-r$, so that $\frac{(m+1)}{2 m}(1+\eta)>\frac{(m+1)}{2 m}(1+\xi-r)=r$. Thus, for every $t \geq 0$, we find that $g(t)-1-\eta \leq(g(t)-1-\eta)-(f(t)-1-\xi)=w_{1}(t)-v_{1}(t)<r<\frac{(m+1)}{2 m}(1+\eta)$, i.e. $g(t)<\frac{(3 m+1)}{2 m}(1+\eta)$. From Lemma 3.4(5), we deduce that $g^{\prime \prime}$ never vanishes over $[0, \infty)$, and therefore $g^{\prime \prime}(t)<0$ for all $t \geq 0$. Using Remark 3.3, we obtain $w_{3}(t)<0$ for all $t \geq 0$, so that $(\mu, w, \eta) \in \Sigma$ and, consequently, $\Sigma$ is both open and closed in $\mathcal{C}_{+}$. The connectedness of $\mathcal{C}_{+}$concludes the proof.

(3) The proof is similar to the previous one, except for the case $m \in\left[-\frac{1}{2},-\frac{1}{3}\right]$ which is this time to be treated together with the case $m \in\left(-1,-\frac{1}{2}\right)$.

\subsection{Solution branches}

With the preliminary work of Sects. 3.1 and 3.2, we are now able to use Proposition 2.7 and obtain an existence result. In the proof, some of our estimates will rely upon the study of an integral formulation of (3.6) which is carried over in Appendix B. In order for the integral equation considered therein to be well-defined (as an asymptotic formulation of our problem), we need the exponent $\alpha>0$ not to be too small. For this reason, we replace (3.15) by the following condition.

$$
\frac{1}{4}(1+\xi)<\alpha(\xi, \lambda)<\frac{1}{2}(1+\xi), \quad \text { for all }(\xi, \lambda) \in U \times \mathbb{R},
$$

where $U$ is given by $(3.20)$.

Theorem 3.8. (Existence of solutions) Let $m \in \mathbb{R} \backslash\{-1\}$ be fixed. Let also $U$ be given by (3.20), $J=\mathbb{R}$, and consider any function $\alpha: U \times J \rightarrow(0, \infty)$ satisfying $\left(G^{\alpha}\right)$ and (3.21). The following statements hold.

(1) If $m \in(-\infty,-1) \cup\left(\frac{1}{3}, \infty\right)$, there exists $-\frac{1}{4} \leq \Lambda<0$ such that for every $\lambda \in[\Lambda, \infty)$ the problem (2.2) has a solution $(\lambda, v, \xi)$ with $v \in C_{0}^{1}\left([0, \infty), \mathbb{R}^{3}\right)$. Moreover, $\lambda v_{3}(t)<0$ for all $t \geq 0$, if $\lambda \neq 0$.

(2) If $m \in\left(-1,-\frac{1}{2}\right)$, there exist $\Lambda_{-}<0<\Lambda_{+}$such that for every $\lambda \in\left(\Lambda_{-}, \Lambda_{+}\right]$the problem $(2.2)$ has a solution $(\lambda, v, \xi)$ with $v \in$ $C_{0}^{1}\left([0, \infty), \mathbb{R}^{3}\right)$. Moreover, $\lambda v_{3}(t)<0$ for all $t \geq 0$, if $\lambda \neq 0$.

(3) If $m \in\left[-\frac{1}{2}, \frac{1}{3}\right]$, there exists $\Lambda<0$ such that for every $\lambda \in(\Lambda, \infty)$ the problem $(2.2)$ has a solution $(\lambda, v, \xi)$ with $v \in C_{0}^{1}\left([0, \infty), \mathbb{R}^{3}\right)$.

Moreover, if $m \in\left[-\frac{1}{3}, \frac{1}{3}\right]$ and $\lambda \neq 0$, or if $m \in\left[-\frac{1}{2},-\frac{1}{3}\right)$ and $\lambda<0$, there holds $\lambda v_{3}(t)<0$ for all $t \geq 0$. If $m \in\left[-\frac{1}{2},-\frac{1}{3}\right)$ and $\lambda>0$, then either $\lambda v_{3}(t)<0$ for all $t \geq 0$, or there exists $t^{*} \geq 0$ for which $v_{3}\left(t^{*}\right)=0$ and $\left(t-t^{*}\right) v_{3}(t)<0$ for all $t \neq t^{*}$.

Proof. From Lemma 3.2 and Proposition 3.7(1), the conclusion of Proposition 2.7 holds with $\mathcal{V}=C_{0}^{1}\left([0, \infty), \mathbb{R}^{3}\right)$. Furthermore, from the choice of $U$, the alternatives for $\mathcal{C}_{ \pm}$can be rewritten as follows. 
- If $m \in(-\infty,-1) \cup\left(\frac{1}{3}, \infty\right)$, then $\mathcal{C}_{+}$is unbounded. Also, either $\mathcal{C}_{-}$is unbounded, or $-\frac{(m+1)}{(3 m+1)} \in \overline{P_{\mathbb{R}^{k}}\left(\mathcal{C}_{-}\right)}$.

- If $m \in\left[-\frac{1}{2}, \frac{1}{3}\right]$, then $\mathcal{C}_{+}$is unbounded. Also, either $\mathcal{C}_{-}$is unbounded, or $-1 \in \overline{P_{\mathbb{R}^{k}}\left(\mathcal{C}_{-}\right)}$.

- If $m \in\left(-1,-\frac{1}{2}\right)$, then either $\mathcal{C}_{+}$is unbounded, or $-\frac{(m+1)}{(3 m+1)} \in \overline{P_{\mathbb{R}^{k}}\left(\mathcal{C}_{+}\right)}$. Also, either $\mathcal{C}_{-}$is unbounded, or $-1 \in \overline{P_{\mathbb{R}^{k}}\left(\mathcal{C}_{-}\right)}$.

In order to precise the behaviour of the branches $\mathcal{C}_{ \pm}$, we now prove that they can only be unbounded if $|\lambda| \rightarrow \infty$ or $\xi \rightarrow-1$. For this we fix $\delta>0$ and $\Lambda_{0} \in \mathbb{R}$, and consider $(\lambda, v, \xi) \in \mathcal{C}_{ \pm}$such that $0 \leq \lambda \leq \Lambda_{0}\left(\operatorname{resp} . \Lambda_{0} \leq \lambda \leq 0\right)$ and $\xi>-1+\delta$.

Since $(\lambda, v, \xi)$ satisfies (2.2) with $G$ given by (3.14), it is sufficient to find a bound on $|\xi|$ and $\|v\|_{\infty}$, in order to have a bound on $\left\|v^{\prime}\right\|_{\infty}$. But Lemma 3.5(a) and (b), together with the condition $\xi>-1+\delta$ imply that $|\xi| \leq \kappa$, for some $\kappa=\kappa\left(\Lambda_{0}, \delta\right)>0$, if $m \in(-\infty,-1) \cup\left[-\frac{1}{2}, \infty\right)$. For the remaining case, i.e. $m \in\left(-1,-\frac{1}{2}\right)$, we deduce from our choice of $U$ that $-1+\delta<\xi \leq-\frac{(m+1)}{(3 m+1)}$. Hence $|\xi| \leq 1:=\kappa$, in this case.

Now, since $\lim _{t \rightarrow \infty}|v(t)|=0$ then, either $|v(t)|<1$ for every $t \geq 0$, or there exists $T \geq 0$ such that $|v(T)|=1$, and $|v(t)| \leq 1$, for all $t \geq T$. In the first case, the desired property is proven. In the second situation, we observe that, by Proposition B.4 and (B.7) we have

$$
1=|v(T)| \leq|\beta|\left|\phi_{3}\right| e^{-\left(\frac{1}{2}(1+\xi)-\alpha(\xi, \lambda)\right) T}+\frac{6\left|\phi_{3}\right|\left(1+\left|\frac{2 m}{m+1}\right|\right)}{(1+\xi)^{2}(4 \alpha(\xi, \lambda)-(1+\xi))} e^{-\alpha(\xi, \lambda) T},
$$

where $\beta=\beta(\lambda, v, \xi)$ is given by Proposition B.4(2). Since $(1+\xi)>\delta>0$, and $\alpha$ satisfies $(3.21)$, it follows that $4 \alpha(\xi, \lambda)-(1+\xi)$ and $\frac{1}{2}(1+\xi)-\alpha(\xi, \lambda)$ are bounded away from 0 , independently of $\xi$ and $\lambda$. Moreover, $|\xi| \leq \kappa$, which means that $\left|\phi_{3}\right|$ is bounded by a constant independent of $\xi$. Finally, using Proposition B.5, we can find some $\kappa_{1}=\kappa_{1}\left(\Lambda_{0}, \delta\right)>0$ satisfying $|\beta| \leq \kappa_{1}$. Summarizing, there exist $C, \gamma>0$ depending only upon $\Lambda_{0}$ and $\delta$, for which $1 \leq C e^{-\gamma T}$.

As a consequence, there exists a $T^{*}=T^{*}\left(\Lambda_{0}, \delta\right) \geq 0$ satisfying $|v(t)| \leq 1$, for all $t \geq T^{*}$. But from Lemma 3.5(c) and (d), together with Proposition 3.7(2) and (3), we can find a $\kappa_{2}=\kappa_{2}\left(\Lambda_{0}, \delta\right)>0$ such that $\sup _{t \in\left[0, T^{*}\right]}|v(t)| \leq \kappa_{2}$. Hence $\|v\|_{\infty} \leq \max \left\{1, \kappa_{2}\right\}$, and the property is proven.

For $m \in(-\infty,-1) \cup\left(\frac{1}{3}, \infty\right)$, Lemma $3.5(\mathrm{a})$, and the fact that $\xi>$ $-\frac{(m+1)}{(3 m+1)}>-1$ imply that $\mathcal{C}_{-}$is bounded. On the other hand, if $m \in\left(-1,-\frac{1}{2}\right)$, the same argument as in Lemma 3.5(a) gives $\lambda \leq \frac{1}{4}\left((1+\xi)^{2}-1\right)$. Therefore, the choice of $U$ implies that $\mathcal{C}_{+}$is bounded in this case. To finish the proof, simply notice that the projection of $\mathcal{C}_{ \pm}$along $\lambda$ is an interval, as follows from the connectedness of $\mathcal{C}_{ \pm}$. Furthermore, the sign of $v_{3}$ is given in Proposition 3.7.

Remark 3.9. The estimate upon $\beta$ obtained via the integral equation studied in Appendix B is a crucial argument in the above proof. It allows to control the asymptotic behaviour of the solutions. In some situations, however, we can 
give a shorter proof of the uniform asymptotic decay of the solutions. The argument goes as follows: if $m \in(-1,0],(3.6)$ implies that $f^{\prime \prime}(t) \exp \left(\frac{1}{2} \int_{0}^{t} f(s) d s\right)$ decreases over $[0, \infty)$. Therefore, if $f^{\prime \prime}(t)>0$ and $f(t) \geq(1+\xi)$ for all $t \geq 0$ we may write $0 \leq f^{\prime \prime}(t) \leq f^{\prime \prime}(0) \exp \left(-\frac{1}{2}(1+\xi) t\right)$. Integrating twice this relation, we obtain similar exponential bounds on $f^{\prime}$ and $f-(1+\xi)$, depending upon $\xi$ and $f^{\prime \prime}(0)$ only. Therefore, Lemma 3.5 can be used to obtain bounds in terms of $\lambda$ only (provided $\xi$ is bounded away from -1 ). A similar argument can also be used when $m<-1$ or $m \geq 0$ and $f^{\prime \prime}(t)<0$ for all $t \geq 0$.

\section{Discussion of the results}

Going back to Problem (3.1), (3.2) and setting $h_{\infty}:=\lim _{\tau \rightarrow \infty} h(\tau)\left[=h_{0}(1+\right.$ $\xi)$ ], Theorem 3.8 gives the existence of following solutions.

- In the case $s=1$, which corresponds to $\lambda(m+1)>0$ and $h_{0}(m+1)>0$, we recover some of the results of Brighi et al. [2-4]. For example, when $m<-1$, two solutions are known to exists for $-h_{0}>0$ large enough and $h_{\infty} \neq 0$. Our method gives only a part of the branch of solutions, namely the part with the concave solution, but we don't find concave-convex solutions. When $-\frac{1}{2} \leq m \leq 1$, we obtain the full branch of solutions corresponding to $h_{0}>0$. In this case, there is only one solution.

- In the case $s=-1$, which corresponds to $\lambda(m+1)<0$ and $h_{0}(m+1)<0$, only partial results exist in the literature. With yet another normalization, Liao [13] discusses the case in which $m<-1$ and $h_{0}>0$, and finds two solutions. We are only able to obtain one solution, in this situation. Magyari and Keller [14] deal with the two special cases $m=-\frac{1}{3}$ and $m=1$. For $h_{0}<0$, the authors obtain a unique bounded solution when $h_{0} \leq-\sqrt{6}$ and $m=-\frac{1}{3}$. In the case $m=1$, two solutions are shown to exists for $h_{0}<-2$ and $h_{\infty} \neq 0$, one solution for $h_{0}=-2$ and no solution when $-2<h_{0}<0$. Our method gives only one solution, but the range of $h_{0}$ for which solutions exist is exhausted in each of these special situations. Moreover, we are able to generalize the results of Magyari and Keller and obtain a branch of solutions for all values of $m>-1$ with $h_{0}<0$, and where $h_{\infty}$ covers the interval $(-\infty, 0)$ (see Fig. 2). To the best of our knowledge, this branch has not been reported elsewhere in the literature.

In both cases the asymptotic behaviour of the solutions is controlled as follows. For every $0<\alpha<\frac{1}{2} \frac{h_{\infty}}{h_{0}}$, there exists $\tilde{\kappa}=\tilde{\kappa}\left(m, h_{0}\right)>0$ such that

$$
\left|h(\tau)-h_{\infty}\right|+\left|\frac{h^{\prime}(\tau)}{h_{0}(m+1)}\right|+\left|\frac{h^{\prime \prime}(\tau)}{h_{0}^{2}(m+1)^{2}}\right| \leq \tilde{\kappa} e^{-\alpha s(m+1) h_{0} \tau}, \quad \text { for all } \tau \geq 0
$$

Figure 2 shows the diagrams $(\lambda, \xi)$ and $\left(h_{0}, h_{\infty}\right)$ respectively for the computed solutions in the case $m=-\frac{3}{4}$. The solutions in $\mathcal{C}$ are represented by the thick part of the curve in Fig. 2a. Figure $2 \mathrm{~b}$ shows the corresponding solutions of (3.1) and (3.2). (The diagrams below have been obtained using the continuation software AUTO [6].) 

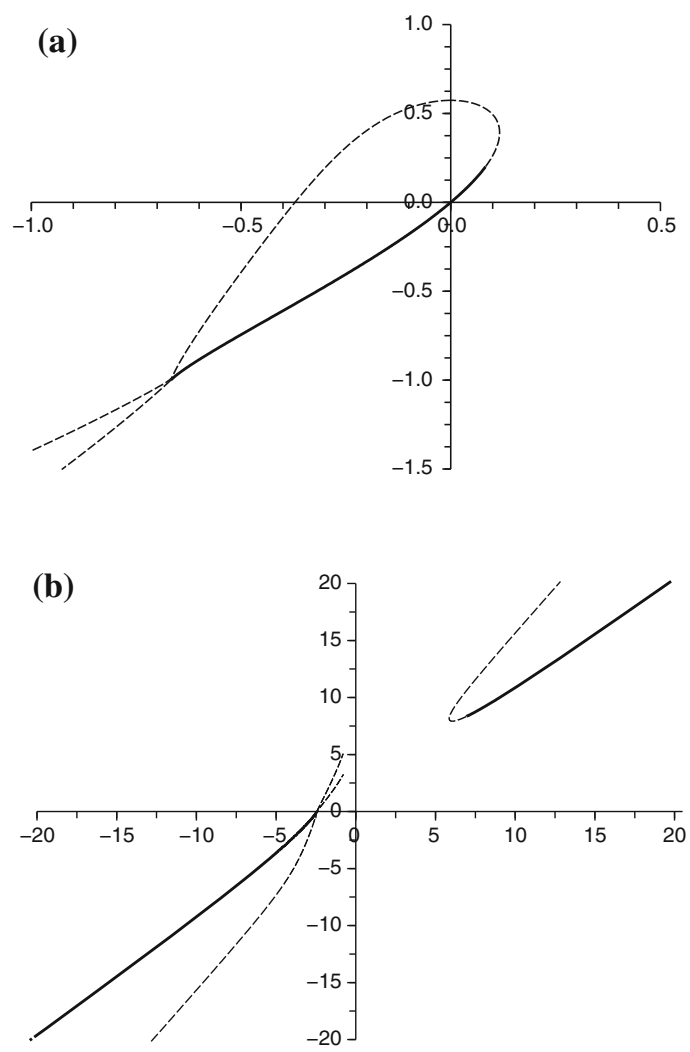

Figure 2. Computed solution branches in the case $m=-\frac{3}{4}$. a Diagram $(\lambda, \xi)$ for solutions of (3.6) and (3.7). b Diagram $\left(h_{0}, h_{\infty}\right)$ for solutions of $(3.1)$ and $(3.2)$

\section{Acknowledgments}

The author would like to thank warmly Prof. C.A. Stuart for his encouragement and support. The helpful remarks of the referees are also gratefully acknowledged.

\section{Appendix A: Proof of Lemmas 3.4 and 3.5}

Proof of Lemma 3.4. (1) First notice that, if $f^{\prime \prime}(t)=0$, then (3.6) gives $f^{\prime \prime \prime}(t)=\frac{m}{m+1}\left(f^{\prime}(t)\right)^{2}$. Moreover, $f^{\prime}(t)=f^{\prime \prime}(t)=0$ implies $f^{\prime} \equiv 0$. Therefore, if $m \neq 0$, all critical points of $\frac{m}{(m+1)} f^{\prime}$ have to be minima. Since $f^{\prime}$ is of class $C^{2}$ over $[0, \infty)$, we conclude that $f^{\prime}$ can have at most one critical point. If this occurs, we have $\frac{m}{(m+1)}\left(t-t^{*}\right) f^{\prime \prime}(t)>0$ for all $t \neq t^{*}$, where $f^{\prime \prime}\left(t^{*}\right)=0$. 
In the case where $m=0,(3.6)$ can be rewritten as

$$
\frac{d}{d t}\left\{f^{\prime \prime}(t) \exp \left(\frac{1}{2} \int_{0}^{t} f(s) d s\right)\right\}=0 .
$$

Therefore, if $f^{\prime \prime}(t)=0$ for some $t \geq 0$, then $f^{\prime \prime} \equiv 0$, on $[0, \infty)$, i.e. $f^{\prime} \equiv 0$. Consequently, $f^{\prime \prime}(t) \neq 0$ for all $t \geq 0$.

(2) Differentiating (3.6), we can write

$$
f^{(i v)}(t)=-\frac{1}{2} f(t) f^{\prime \prime \prime}(t)+\frac{(3 m-1)}{(m+1)} f^{\prime}(t) f^{\prime \prime}(t), \quad \text { for all } t>0 .
$$

From Part (1), there exists some $t^{*} \geq 0$ such that for every $t>t^{*}$, $f^{\prime \prime}(t) \neq 0$. Since $\lim _{t \rightarrow \infty} f^{\prime}(t)=0$, we obtain that $f^{\prime}(t) f^{\prime \prime}(t)>0$, for all $t>t^{*}$. The same argument as in Part (1) (where only the case $m=0$ is replaced by $m=\frac{1}{3}$ ) imply that $f^{\prime \prime \prime}$ can have at most one zero in $\left(t^{*}, \infty\right)$. Hence, there exists $t_{0} \geq t^{*}$ such that $f^{\prime \prime}$ is monotone and of constant sign over $\left(t_{0}, \infty\right)$. Since $\int_{t_{0}}^{+\infty} f^{\prime \prime}(t)=-f^{\prime}\left(t_{0}\right)$, we find that $f^{\prime \prime} \in L^{1}\left(t_{0}, \infty\right)$, and therefore $\lim _{t \rightarrow \infty} f^{\prime \prime}(t)=0$.

(3) By Part (1), $f^{\prime}$ has at most one critical point. Therefore, $f^{\prime}$ is ultimately of constant sign, i.e. $f$ is ultimately monotone.

Now suppose that $m<-1$, or $m \geq 0$. Since $\frac{m}{m+1} \geq 0$, it follows that the only possible critical point of $f^{\prime}$ is a minimum. Next, since $f, f^{\prime}$, and $f^{\prime \prime}$ are ultimately monotone, there exists $t^{*} \geq 0$ such that $f, f^{\prime}, f^{\prime \prime}$, and $f^{\prime \prime \prime}$ have constant sign in $\left(t^{*}, \infty\right)$. We distinguish between two cases: (i) $f^{\prime}\left(t^{*}\right)>0$, and (ii) $f^{\prime}\left(t^{*}\right) \leq 0$.

(i) If $f^{\prime}\left(t^{*}\right)>0$, then $f^{\prime}$ has no critical point, since $\lim _{t \rightarrow \infty} f^{\prime}(t)=0$. Thus, $f^{\prime}(t)>0$ and $f^{\prime \prime}(t)<0$ for all $t \geq 0$, so that $f$ increases strictly over $[0, \infty)$, and $0<f(0)<\lim _{t \rightarrow \infty} f(t) \leq+\infty$. To see that this limit is finite, we argue as follows. Remark that (3.6) can be rewritten as

$$
\frac{d}{d t}\left\{f^{\prime \prime}(t) \exp \left(\frac{1}{2} \int_{0}^{t} f(s) d s\right)\right\}=\frac{m}{m+1}\left(f^{\prime}(t)\right)^{2} \exp \left(\frac{1}{2} \int_{0}^{t} f(s) d s\right) .
$$

Since $\frac{m}{m+1} \geq 0, f^{\prime \prime}(t)<0$, and $f(t)>f(0)>0$, it follows that

$$
\left(-f^{\prime \prime}(0)\right) \exp \left(-\frac{1}{2} f(0) t\right) \geq\left(-f^{\prime \prime}(t)\right)
$$

for all $t>0$. Integrating the above relation twice over $(t, \infty)$ and evaluating at $t=0$, we find

$$
\lim _{t \rightarrow \infty} f(t) \leq f(0)-4 f^{\prime \prime}(0)<+\infty .
$$

(ii) On the other hand, if $f^{\prime}\left(t^{*}\right) \leq 0$, then $f^{\prime}(t)<0$, for all $t>t^{*}$, since $f^{\prime}$ can only have a minimum in $[0, \infty)$, and $\lim _{t \rightarrow \infty} f^{\prime}(t)=0$. As a consequence, $-\infty \leq \lim _{t \rightarrow \infty} f(t)<f\left(t^{*}\right)$. Moreover, $f^{\prime \prime}(t)>0$ and $f^{\prime \prime \prime}(t)<0$ for all $t>t^{*}$ which, together with (3.6), gives

$$
f(t)=\frac{\frac{2 m}{m+1}\left(f^{\prime}(t)\right)^{2}-2 f^{\prime \prime \prime}(t)}{f^{\prime \prime}(t)}>0,
$$


for all $t>t^{*}$. Thus, $0 \leq \lim _{t \rightarrow \infty} f(t)<+\infty$. It remains to see that $f(t)>0$, for all $t \geq 0$. We have already shown that this property holds on $\left[t^{*}, \infty\right)$. Now, either $f^{\prime}(t)<0$ for all $t>0$, in which case $f(t)>\lim _{s \rightarrow \infty} f(s) \geq 0$ for all $t \geq 0$, or there exists $0<t_{0} \leq t^{*}$ such that $f^{\prime}\left(t_{0}\right)=0$. In this case $f^{\prime}(t)>0$ for $0 \leq t<t_{0}$ and $f^{\prime}(t)<0$ for $t>t_{0}$. Hence $f(t)>f(0)>0$ on $\left[0, t_{0}\right], f(t) \geq f\left(t^{*}\right)$ on $\left(t_{0}, t^{*}\right)$, and the conclusion follows.

(4) Follows from (3.6), after an integration by parts.

(5) First assume that $m \notin\left[-\frac{1}{3}, \frac{1}{3}\right]$ (recall that $m \neq-1$, by hypothesis). In this case, (3.17) evaluated at $t=t^{*}$ gives

$$
-\frac{(m+1)}{(3 m+1)} f\left(t^{*}\right) f^{\prime}\left(t^{*}\right)=\int_{t^{*}}^{+\infty}\left(f^{\prime}(s)\right)^{2} d s .
$$

Since $f^{\prime \prime}$ has constant sign on $\left(t^{*}, \infty\right)$ and $\lim _{t \rightarrow \infty} f^{\prime}(t)=0$, we know that $0<\left(f^{\prime}(s)\right)^{2}<f^{\prime}\left(t^{*}\right) f(s)$ for all $s>t^{*}$. Therefore,

$$
-\frac{(m+1)}{(3 m+1)} f\left(t^{*}\right) f^{\prime}\left(t^{*}\right)<f^{\prime}\left(t^{*}\right)\left(1+\xi-f\left(t^{*}\right)\right) .
$$

Now, observe that $f^{\prime}\left(t^{*}\right)>0$ when $f^{\prime \prime \prime}\left(t^{*}\right)<0$, and $f^{\prime}\left(t^{*}\right)<0$ when $f^{\prime \prime \prime}\left(t^{*}\right)>$ 0 , since $\lim _{t \rightarrow \infty} f^{\prime}(t)=0$. Consequently, (3.6) implies that $\frac{m}{m+1} f^{\prime}\left(t^{*}\right)<0$, which, together with the preceding inequality, gives (3.19).

It remains to show that $f^{\prime \prime}$ cannot vanish in $[0, \infty)$, when $m \in\left[-\frac{1}{3}, \frac{1}{3}\right]$. First assume that $m \in\left[-\frac{1}{3}, 0\right)$, and that $f^{\prime \prime}$ vanishes at some $t^{*} \geq 0$. In this case, it follows from (3.6), that $f^{\prime}\left(t^{*}\right)$ is a maximum, and therefore $f^{\prime \prime}(t)>0$ for all $0 \leq t<t^{*}$, and $f^{\prime}\left(t^{*}\right)>0$. Using (3.17), we find that $f^{\prime}(t) f(t)<0$, for all $0 \leq t \leq t^{*}$. In particular, this implies that $f^{\prime}$ does not vanish in $\left[0, t^{*}\right)$, which, in turn, gives $f(t)<0$ for all $0 \leq t<t^{*}$. Since $f(0)>0$, the only possible conclusion is that $t^{*}=0$. But, in this case, (3.17) implies that $f(0) f^{\prime}(0) \leq 0$, and therefore, necessarily $f^{\prime}(0)=0$, i.e. $f^{\prime} \equiv 0$. Since this case is excluded, we obtain that $f^{\prime \prime}$ never vanishes in $[0, \infty)$.

Now consider the case where $m \in\left[0, \frac{1}{3}\right]$, and recall that the result for $m=0$ has been proven in part (1). For $m \in\left(0, \frac{1}{3}\right)$ suppose, by contradiction, there were some $t^{*} \geq 0$ satisfying $f^{\prime \prime}\left(t^{*}\right)=0$. Part (1) would give $f^{\prime \prime}(t)>0$, and therefore $f^{\prime}(t)<0$, for all $t>t^{*}$. From (A.1), we know that $f^{\prime \prime \prime}$ vanishes at most once in $\left(t^{*}, \infty\right)$, and that, in this case, $f^{\prime \prime \prime}(t)>0$ for all $t>t_{0}$, if $f^{\prime \prime \prime}\left(t_{0}\right)=0$. Since, $\lim _{t \rightarrow \infty} f^{\prime \prime}(t)=0$, we conclude that $f^{\prime \prime \prime}(t)<0$ for all $t>t^{*}$, and therefore $f^{\prime \prime \prime}\left(t^{*}\right) \geq 0$, by continuity. But remark that (3.6) gives $f^{\prime \prime \prime}\left(t^{*}\right)>0$ (since $\left.f^{\prime} \not \equiv 0\right)$. This contradiction proves the desired result.

It remains to see what happens when $m=\frac{1}{3}$. In this case, (A.1) can be rewritten as

$$
\frac{d}{d t}\left\{f^{\prime \prime \prime}(t) \exp \left(\frac{1}{2} \int_{0}^{t} f(s) d s\right)\right\}=0, \quad \text { for all } t \geq 0 .
$$

Consequently, $f^{\prime \prime \prime}$ never vanishes in $[0, \infty)$, (unless $f^{\prime} \equiv 0$, which is excluded here) and therefore, the same is true for $f^{\prime \prime}$, since $\lim _{t \rightarrow \infty}$ $f^{\prime \prime}(t)=0$. 
Proof of Lemma 3.5. (a) We deduce from the identity (3.17) that $\frac{d}{d t}\left\{f^{\prime}(t)+\right.$ $\left.\frac{1}{4}(f(t))^{2}\right\} \leq 0$ for all $t \geq 0$. Therefore, $\lambda+\frac{1}{4} \geq \frac{1}{4}(1+\xi)^{2}$, which proves the result.

(b) From (3.18) we see that $f(t) f^{\prime \prime}(t)-\frac{1}{2}\left(f^{\prime}(t)\right)^{2}+\frac{1}{2}(f(t))^{2} f^{\prime}(t) \leq 0$ for all $t \geq 0$. Integrating over $(0, \infty)$, and using $(3.17)$ at $t=0$, we obtain

$$
\begin{gathered}
\frac{1}{6}(1+\xi)^{3} \leq \lambda+\frac{1}{6}+\frac{3}{2} \int_{0}^{+\infty}\left(f^{\prime}(s)\right)^{2} d s \\
=\lambda+\frac{1}{6}-\frac{3(m+1)}{(3 m+1)}\left(f^{\prime \prime}(0)+\frac{1}{2} \lambda\right) .
\end{gathered}
$$

Moreover, if we evaluate (3.18) at $t=0$, we obtain $f^{\prime \prime}(0)+\frac{1}{2} \lambda \leq \frac{1}{2} \lambda^{2}$, and the result is proven.

(c) First assume that $f^{\prime \prime}(t)>0$ for all $t \geq 0$, and $\xi>-1$. In this case, $\lambda \leq f^{\prime}(t)<0$ and $0<(1+\xi)<f(t) \leq 1$, for all $t \geq 0$. Furthermore, if $m \in(-\infty,-1) \cup\left[-\frac{1}{3},+\infty\right),(3.17)$ gives $f^{\prime \prime}(t) \leq-\frac{1}{2} f(t) f^{\prime}(t) \leq-\frac{1}{2} \lambda$, for all $t \geq 0$. Since $-\frac{1}{2} \lambda \leq \frac{1}{2}|\lambda|+\frac{(4|m|+1)}{2|m+1|} \lambda^{2}$, the result is true in this case.

It remains to see what happens when $m \in\left(-1,-\frac{1}{3}\right)$. By Lemma $3.4(2), f^{\prime \prime}$ has at most one critical point in $[0, \infty)$, and $\lim _{t \rightarrow \infty} f^{\prime \prime}(t)=0$. But from (A.1), we see that $f^{\prime \prime}$ can only have a minimum. Therefore, $f^{\prime \prime}$ has no critical point and $0<f^{\prime \prime}(t) \leq f^{\prime \prime}(0)$ for all $t \geq 0$. Next, observe that when $m \in\left(-1,-\frac{1}{2}\right),(3.17)$ and $(3.18)$ evaluated at $t=0$ imply

$$
\begin{aligned}
& -\frac{(3 m+1)}{2(2 m+1)}\left(f^{\prime \prime}(0)-\frac{1}{2} \lambda^{2}+\frac{1}{2} \lambda\right) \\
& \geq \frac{(3 m+1)}{2(m+1)} \int_{0}^{+\infty}\left(f^{\prime}(s)\right)^{2} d s=-\left(f^{\prime \prime}(0)+\frac{1}{2} \lambda\right) .
\end{aligned}
$$

Hence, $f^{\prime \prime}(0) \leq-\frac{1}{2} \lambda-\frac{(3 m+1)}{2(m+1)} \lambda^{2} \leq \frac{1}{2}|\lambda|+\frac{4|m|+1}{2|m+1|} \lambda^{2}$. On the other hand, when $m \in\left[-\frac{1}{2},-\frac{1}{3}\right)$, we obtain $f^{\prime \prime}(0) \leq-\frac{1}{2} \lambda+\frac{1}{2} \lambda^{2} \leq \frac{1}{2}|\lambda|+\frac{4|m|+1}{2|m+1|} \lambda^{2}$, by (3.18) at $t=0$, and the desired result holds.

Now we turn to the situation where $f^{\prime \prime}(t)<0$ for all $t \geq 0$. Under this condition, we have $0<f^{\prime}(t) \leq \lambda$ and $1 \leq f(t)<(1+\xi)$, for all $t \geq 0$. If $m \in(-\infty,-1) \cup\left[-\frac{1}{3},+\infty\right)$, the same argument as in the case $m \in\left(-1,-\frac{1}{2}\right)$ above shows that $f^{\prime \prime}$ has no critical point, and $-\frac{1}{2} \lambda-$ $\frac{(3 m+1)}{2(m+1)} \lambda^{2} \leq f^{\prime \prime}(0) \leq f^{\prime \prime}(t)<0$ for all $t \geq 0$. Thus the result holds in this case.

For $m \in\left(-1,-\frac{1}{3}\right)$, Lemma 3.4(2) also implies that $f^{\prime \prime}$ has at most one critical point in $[0, \infty)$. Hence, we find that either $f^{\prime \prime}(0) \leq f^{\prime \prime}(t)<0$, or $f^{\prime \prime}(\bar{t}) \leq f^{\prime \prime}(t)<0$ for all $t \geq 0$, where $\bar{t} \geq 0$ satisfies $f^{\prime \prime \prime}(\bar{t})=0$. In the first case, we evaluate (3.17) at $t=0$ and find $f^{\prime \prime}(0) \geq \frac{1}{2} \lambda$. In the second case, (3.6) at $t=\bar{t}$ gives $f^{\prime \prime}(\bar{t}) \geq \frac{2 m}{(m+1)} \lambda^{2}$. Since $\frac{2|m|}{|m+1|} \lambda^{2} \leq$ $\frac{1}{2}|\lambda|+\frac{(4|m|+1)}{2|m+1|} \lambda^{2}$, we are done. 
(d) From Lemma 3.4(1), $f^{\prime \prime}(t)>0$ for all $t \in\left[0, t^{*}\right]$ and $f^{\prime \prime}(t)<0$ for all $t>t^{*}$. Since $\lambda>0$, we obtain $0<f^{\prime}(t) \leq f^{\prime}\left(t^{*}\right)$ and $1 \leq f(t)<(1+\xi)$, for all $t \geq 0$. By Lemma $3.4(2)$, we have $\lim _{t \rightarrow \infty} f^{\prime \prime}(t)=0$, and it follows that there exists a unique $t_{0} \geq 0$ for which $f^{\prime \prime \prime}\left(t_{0}\right)=0$. Thus, $f^{\prime \prime}\left(t_{0}\right) \leq f^{\prime \prime}(t) \leq f^{\prime \prime}(0)$ for every $t \geq 0$. Using (3.18) at $t=0$, we obtain $f^{\prime \prime}(0) \leq \frac{1}{2}\left(\lambda^{2}-\lambda\right) \leq \frac{1}{2}\left(f^{\prime}\left(t^{*}\right)\right)^{2}$. Also, from (3.6), it follows that $f^{\prime \prime}\left(t_{0}\right) \geq \frac{2 m}{m+1}\left(f^{\prime}\left(t^{*}\right)\right)^{2} \geq-2\left(f^{\prime}\left(t^{*}\right)\right)^{2}$. Finally, integrating (3.17) over $\left[t^{*}, \infty\right)$, we deduce that $f^{\prime}\left(t^{*}\right)+\frac{1}{4}\left(1-(1+\xi)^{2}\right) \leq 0$, which concludes the proof.

\section{Appendix B: An integral equation}

In this section, we wish to study the asymptotic behaviour of the solutions of (3.6) and (3.7). For this purpose, we rewrite the problem as an integral equation, and show that the latter has a unique solution among functions which decay sufficiently fast at infinity. The arguments presented below are closely related to those developed by McLeod [15] when proving the existence of a solution to the swirling flow problem.

We begin with the definition of a particular function space.

Definition B.1. For $T \geq 0$ and $\alpha>0$, we set

$$
Y_{T}^{\alpha}:=\left\{u \in C\left([T, \infty), \mathbb{R}^{3}\right): \sup _{t \geq T}\left|e^{\alpha t} u(t)\right|<+\infty\right\} .
$$

This space is equipped with the norm $\|\cdot\|_{\alpha, T}$ defined by

$$
\|u\|_{\alpha, T}:=\sup _{t \geq T}\left|e^{\alpha t} u(t)\right|, \text { for all } u \in Y_{T}^{\alpha} .
$$

$\left(Y_{T}^{\alpha},\|\cdot\|_{\alpha, T}\right)$ is a Banach space.

Lemma B.2. Let $m \in \mathbb{R} \backslash\{-1\}, \xi>-1, T \geq 0$, and $\frac{1}{4}(1+\xi)<\alpha<\frac{1}{2}(1+\xi)$ be given. For $w \in \mathbb{R}^{3}$, we let

$$
N_{3}(w):=\frac{1}{2} w_{1} w_{3}-\frac{m}{m+1} w_{2}^{2} .
$$

Then, for every $u \in Y_{T}^{\alpha}$ and for all $t \geq T$ the generalized integrals

$$
\begin{gathered}
I_{1}(t)=\int_{t}^{+\infty}(t-\tau) N_{3}(u(\tau)) d \tau, \quad I_{2}(t)=\int_{t}^{+\infty} N_{3}(u(\tau)) d \tau \\
\quad \text { and } \quad I_{3}(t)=\int_{t}^{+\infty} e^{\frac{1}{2}(1+\xi)(\tau-t)} N_{3}(u(\tau)) d \tau
\end{gathered}
$$

converge absolutely. Moreover,

(1) $I_{i} \in C_{0}^{1}([T, \infty), \mathbb{R})$, for $i=1,2,3, I_{1}^{\prime}(t)=I_{2}(t), I_{2}^{\prime}(t)=-N_{3}(u(t))$, and $I_{3}^{\prime}(t)=-N_{3}(u(t))-\frac{1}{2}(1+\xi) I_{3}(t)$, for all $t \geq T$.

$$
\begin{aligned}
& \left|I_{1}(t)\right| \leq\left(\frac{1}{2}+\left|\frac{m}{m+1}\right|\right) \frac{\|u\|_{\alpha, T}^{2}}{4 \alpha^{2}} e^{-2 \alpha t},\left|I_{2}(t)\right| \leq\left(\frac{1}{2}+\left|\frac{m}{m+1}\right|\right) \frac{\|u\|_{\alpha, T}^{2}}{2 \alpha} e^{-2 \alpha t}, \\
& \text { and }\left|I_{3}(t)\right| \leq\left(\frac{1}{2}+\left|\frac{m}{m+1}\right|\right) \frac{2\|u\|_{\alpha, T}^{2}}{(4 \alpha-(1+\xi))} e^{-2 \alpha t}, \text { for all } t \geq T
\end{aligned}
$$


Proof. For $b \geq T$, we have

$$
\begin{aligned}
\int_{t}^{b}\left|(t-\tau) N_{3}(u(\tau))\right| d \tau \leq & \int_{t}^{b}(\tau-t) e^{-2 \alpha \tau}\left|N_{3}\left(e^{\alpha \tau} u(\tau)\right)\right| d \tau \\
\leq & \left(\frac{1}{2}+\left|\frac{m}{m+1}\right|\right)\|u\|_{\alpha, T}^{2} \\
& \times\left\{\frac{(t-b)}{2 \alpha} e^{-2 \alpha b}+\frac{1}{4 \alpha^{2}}\left[e^{-2 \alpha t}-e^{-2 \alpha b}\right]\right\},
\end{aligned}
$$

for all $T \leq t \leq b$. Thus,

$$
\lim _{b \rightarrow+\infty} \int_{t}^{b}\left|(t-\tau) N_{3}(u(\tau))\right| d \tau \leq\left(\frac{1}{2}+\left|\frac{m}{m+1}\right|\right) \frac{\|u\|_{\alpha, T}^{2}}{4 \alpha^{2}} e^{-2 \alpha t}<+\infty,
$$

for all $t \geq T$. Similarly, we find that

$$
\lim _{b \rightarrow+\infty} \int_{t}^{b}\left|N_{3}(u(\tau))\right| d \tau \leq\left(\frac{1}{2}+\left|\frac{m}{m+1}\right|\right) \frac{\|u\|_{\alpha, T}^{2}}{2 \alpha} e^{-2 \alpha t}<+\infty
$$

and

$$
\lim _{b \rightarrow+\infty} \int_{t}^{b} e^{\frac{1}{2}(1+\xi)(\tau-t)}\left|N_{3}(u(\tau))\right| d \tau \leq\left(\frac{1}{2}+\left|\frac{m}{m+1}\right|\right) \frac{2\|u\|_{\alpha, T}^{2}}{(4 \alpha-(1+\xi))} e^{-2 \alpha t}<+\infty,
$$

for all $t \geq T$, since $\alpha>\frac{1}{4}(1+\xi)>0$ and $u \in Y_{T}^{\alpha}$. Therefore, the generalized integrals above converge absolutely on $[T, \infty)$ and the estimates in part $(2)$ are proven.

From the theory of generalized Riemann integrals, (see for example [16, Theorems 9-2, 11-1, and 11-4]) it follows that $I_{i} \in C^{1}([T, \infty), \mathbb{R})$, $\lim _{t \rightarrow \infty} I_{i}(t)=0$, for $i=1,2,3$, and the derivatives are given by $I_{1}^{\prime}(t)=$ $-I_{2}(t), I_{2}^{\prime}(t)=-N_{3}(u(t))$ and $I_{3}^{\prime}(t)=-N_{3}(u(t))-\frac{1}{2}(1+\xi) I_{3}(t)$, for all $t \geq T$. Finally, $\lim _{t \rightarrow \infty} I_{i}^{\prime}(t)=0$, so that $I_{i} \in C_{0}^{1}([T, \infty), \mathbb{R})$, for $i=1,2,3$.

Now we study the existence and uniqueness of solutions to a particular integral equation in $Y_{T}^{\alpha}$, using the Banach fixed-point Theorem.

Proposition B.3. Let $m \in \mathbb{R} \backslash\{-1\}, \xi>-1, \frac{1}{4}(1+\xi)<\alpha<\frac{1}{2}(1+\xi)$, and $\beta \in \mathbb{R}$ be given. For $T \geq 0$ and $u \in Y_{T}^{\alpha}$, let

$$
\mathcal{H}(u)(t):=\beta e^{-\frac{1}{2}(1+\xi) t} \phi_{3}+\frac{2}{1+\xi} I_{1}(t) \phi_{1}-\frac{4}{(1+\xi)^{2}} I_{2}(t) \phi_{2}+\frac{4}{(1+\xi)^{2}} I_{3}(t) \phi_{3},
$$

for all $t \geq T$, where $I_{1}, I_{2}$, and $I_{3}$ are defined in Lemma B.2, and where $\phi_{1}=(1,0,0), \phi_{2}=\left(1,-\frac{1}{2}(1+\xi), 0\right)$, and $\phi_{3}=\left(1,-\frac{1}{2}(1+\xi), \frac{1}{4}(1+\xi)^{2}\right)$.

Then $\mathcal{H}: Y_{T}^{\alpha} \rightarrow Y_{T}^{\alpha}$ is well-defined, and the following statements hold.

Let $K>1$ be given, and set

$$
\rho(T)=\frac{(1+\xi)^{2}(4 \alpha-(1+\xi))}{12 K\left|\phi_{3}\right|\left(1+\left|\frac{2 m}{m+1}\right|\right)} e^{\alpha T} .
$$

(1) For all $u, v \in Y_{T}^{\alpha}$ with $\|u\|_{\alpha, T},\|v\|_{\alpha, T} \leq \rho(T)$,

$$
|\mathcal{H}(u)(t)-\mathcal{H}(v)(t)| \leq \frac{1}{K} e^{-\alpha(2 t-T)}\|u-v\|_{\alpha, T}, \quad \text { for all } t \geq T,
$$


and therefore

$$
\|\mathcal{H}(u)-\mathcal{H}(v)\|_{\alpha, T} \leq \frac{1}{K}\|u-v\|_{\alpha, T} .
$$

(2) Let $T_{0}=\frac{2}{1+\xi} \ln \left(\frac{24 K^{2}|\beta|\left|\phi_{3}\right|^{2}\left(1+\left|\frac{2 m}{m+1}\right|\right)}{(2 K-1)(1+\xi)^{2}(4 \alpha-(1+\xi))}\right)$. If $T \geq T_{0}$, then $\mathcal{H}$ is a strict contraction in $\left\{u \in Y_{T}^{\alpha}:\|u\|_{\alpha, T} \leq \rho(T)\right\}$, and the equation $\mathcal{H}(u)=u$ has a unique solution $\widehat{u}$ such that $\|\widehat{u}\|_{\alpha, T} \leq \rho(T)$. Moreover,

$$
\lim _{k \rightarrow+\infty}\left\|\mathcal{H}^{k}(0)-\widehat{u}\right\|_{\alpha, T}=0 .
$$

Proof. Let $u \in Y_{T}^{\alpha}$, it follows from Lemma B.2 (1) that $\mathcal{H}(u) \in C_{0}^{1}\left([T, \infty), \mathbb{R}^{3}\right)$. Moreover, the bounds in Part (2) of the same lemma imply

$$
\begin{aligned}
\left|e^{\alpha t} \mathcal{H}(u)(t)\right| \leq & |\beta| e^{-\left(\frac{1}{2}(1+\xi)-\alpha\right) t}\left|\phi_{3}\right|+\left(\frac{1}{2}+\left|\frac{m}{m+1}\right|\right) e^{-\alpha t}\|u\|_{\alpha, T}^{2}\left\{\frac{\left|\phi_{1}\right|}{2 \alpha^{2}(1+\xi)}\right. \\
& \left.+\frac{2\left|\phi_{2}\right|}{\alpha(1+\xi)^{2}}+\frac{8\left|\phi_{3}\right|}{(4 \alpha-(1+\xi))(1+\xi)^{2}}\right\} \\
\leq & |\beta|\left|\phi_{3}\right| e^{-\left(\frac{1}{2}(1+\xi)-\alpha\right) t}+\left(\frac{1}{2}+\left|\frac{m}{m+1}\right|\right) \frac{12\left|\phi_{3}\right|\|u\|_{\alpha, T}^{2}}{(1+\xi)^{2}(4 \alpha-(1+\xi))} e^{-\alpha t},
\end{aligned}
$$

for all $t \geq T$, using the fact that $\frac{1}{4}(1+\xi)<\alpha<\frac{1}{2}(1+\xi)$. Hence, $\mathcal{H}(u) \in Y_{T}^{\alpha}$, and

$$
\|\mathcal{H}(u)\|_{\alpha, T} \leq|\beta|\left|\phi_{3}\right| e^{-\left(\frac{1}{2}(1+\xi)-\alpha\right) T}+\frac{6\left|\phi_{3}\right|\left(1+\left|\frac{2 m}{m+1}\right|\right)\|u\|_{\alpha, T}^{2}}{(1+\xi)^{2}(4 \alpha-(1+\xi))} e^{-\alpha T} .
$$

(1) Let $K>1$ and consider $u, v \in Y_{T}^{\alpha}$, satisfying $\|u\|_{\alpha, T},\|v\|_{\alpha, T} \leq \rho(T)$. First, we remark that for $w, z \in \mathbb{R}^{3}$,

$$
\left|N_{3}(w)-N_{3}(z)\right| \leq\left(\frac{1}{2}+\left|\frac{m}{m+1}\right|\right)(|w|+|z|)|w-z| .
$$

Therefore, $\left|N_{3}(u(\tau))-N_{3}(v(\tau))\right| \leq 2\left(\frac{1}{2}+\left|\frac{m}{m+1}\right|\right) \rho(T) e^{-2 \alpha \tau}\|u-v\|_{\alpha, T}$, for every $\tau \geq T$. Consequently, for all $t \geq T$, we have

$$
\begin{aligned}
|\mathcal{H}(u)(t)-\mathcal{H}(v)(t)| \leq & \left(1+\left|\frac{2 m}{m+1}\right|\right) \rho(T)\|u-v\|_{\alpha, T} \\
& \times\left\{\frac{2\left|\phi_{1}\right|}{(1+\xi)} \int_{t}^{+\infty}(t-\tau) e^{-2 \alpha \tau} d \tau\right. \\
& +\frac{4\left|\phi_{2}\right|}{(1+\xi)^{2}} \int_{t}^{+\infty} e^{-2 \alpha \tau} d \tau \\
& \left.\left.+\frac{4\left|\phi_{3}\right|}{(1+\xi)^{2}} \int_{t}^{+\infty} e^{\frac{1}{2}(1+\xi)(\tau-t)} e^{-2 \alpha \tau} d \tau\right)\right\}, \\
\leq & \frac{12\left|\phi_{3}\right|\left(1+\left|\frac{2 m}{m+1}\right|\right) \rho(T)}{(1+\xi)^{2}(4 \alpha-(1+\xi))} e^{-2 \alpha t}\|u-v\|_{\alpha, T} \\
= & \frac{1}{K} e^{-\alpha(2 t-T)}\|u-v\|_{\alpha, T} .
\end{aligned}
$$

This proves (B.5), and (B.6) follows at once from the definition of $\|\cdot\|_{\alpha, T}$. 
(2) To begin with, let us remark that (B.7) can be rewritten as

$$
\|\mathcal{H}(u)\|_{\alpha, T} \leq|\beta|\left|\phi_{3}\right| e^{-\left(\frac{1}{2}(1+\xi)-\alpha\right) T}+\frac{\|u\|_{\alpha, T}^{2}}{2 K \rho(T)} .
$$

Therefore, if $T \geq T_{0}$, and $\|u\|_{\alpha, T} \leq \rho(T)$, then

$$
\|\mathcal{H}(u)\|_{\alpha, T} \leq \frac{(2 K-1) \rho\left(T_{0}\right)}{2 K}+\frac{\|u\|_{\alpha, T}^{2}}{2 K \rho(T)} \leq \rho(T) .
$$

Now, since (B.6) holds, it follows that $\mathcal{H}$ maps the set $\left\{u \in Y_{T}^{\alpha}\right.$ : $\left.\|u\|_{\alpha, T} \leq \rho\right\}$ into itself and is a strict contraction in this set, for $K>1$. Hence, the hypotheses of the Banach fixed-point Theorem hold and there exists a unique $\widehat{u}$ in this ball satisfying $\mathcal{H}(\widehat{u})=\widehat{u}$. Moreover, $\widehat{u}=\lim _{k \rightarrow+\infty} \mathcal{H}^{k}(0)$ in $Y_{T}^{\alpha}$.

The following result relates the solution $\widehat{u}$ of the integral equation $\mathcal{H}(u)=u$ given in Proposition B.3 and the solutions $(\lambda, v, \xi)$ of $(2.2)$. As we will see, both of them coincide, at least on the domain $\left[T_{0}, \infty\right)$.

We recall some notation: let $\mathcal{C}$ denote the connected component of solutions of $(2.2)$ in $\mathbb{R} \times C_{0}^{1}\left([0, \infty), \mathbb{R}^{3}\right) \times U$, which contains $(0,0,0)$, and where $U$ is given by $(3.20)$. Moreover, $\alpha$ is now a function of $(\xi, \lambda)$ which satisfies $\left(\mathrm{G}^{\alpha}\right)$ and the condition (3.21).

Proposition B.4. Let $m \in \mathbb{R} \backslash\{-1\}$ be fixed, and consider $(\lambda, v, \xi) \in \mathcal{C}$. Let $u(t)=e^{-\alpha(\xi, \lambda) t} v(t)$, for all $t \geq 0$.

(1) For all $T \geq 0, u \in Y_{T}^{\alpha(\xi, \lambda)}$ and $\|u\|_{\alpha(\xi, \lambda), T}=\sup _{t \geq T}|v(t)|$.

(2) There exists $\beta=\beta(\lambda, v, \xi) \in \mathbb{R}$ such that $u(t)=\mathcal{H}(u(t))$, for all $t \geq 0$.

(3) For every $K>1$, we have $u(t)=\widehat{u}(t)$ for all $t \geq T_{0}$, where $T_{0}$ and $\widehat{u}$ are given by Proposition B.3(2) with $\alpha=\alpha(\xi, \lambda)$.

Proof. (1) Follows from the definition of $u$ and the fact that $v \in C_{0}^{1}$ $\left([0, \infty), \mathbb{R}^{3}\right)$.

(2) Observe that, since $(\lambda, v, \xi)$ is a solution of $(2.2)$, then $(\lambda, u, \xi)$ solves the system (1.2). Now let $w=u-\mathcal{H}(u)$, where $\mathcal{H}$ is given by (B.2), and $\beta \in \mathbb{R}$ is arbitrary. By Lemma B.2, $w \in C_{0}^{1}\left([0, \infty), \mathbb{R}^{3}\right)$, and

$$
w^{\prime}(t)=u^{\prime}(t)+\frac{1}{2}(1+\xi) \beta e^{-\frac{1}{2}(1+\xi) t} \phi_{3}-\frac{2}{1+\xi} I_{2}(t) \phi_{1}-N(u(t))+\frac{2}{1+\xi} I_{3}(t) \phi_{3},
$$

for all $t \geq 0$, since $N(u(t))=\left(0,0, N_{3}(u(t))\right)$, where $N(u(t))$ is given by (3.13). Hence, since $A(\xi) \phi_{1}=(0,0,0), A(\xi) \phi_{2}=\frac{1}{2}(1+\xi) \phi_{1}$, and $A(\xi) \phi_{3}=\frac{1}{2}(1+\xi) \phi_{3}$, we obtain that $w^{\prime}(t)+A(\xi) w(t)=0$, for all $t \geq 0$. A fundamental set of solutions to the linear system is given by $\left\{(1,0,0),(t, 1,0), e^{-\frac{1}{2}(1+\xi) t} \phi_{3}\right\}$. Using the fact that $\lim _{t \rightarrow \infty} w(t)=0$, we conclude that there exists $\widehat{\beta} \in \mathbb{R}$ such that $w(t)=\widehat{\beta} e^{-\frac{1}{2}(1+\xi) t} \phi_{3}$, and the conclusion follows by replacing $\beta$ with $\beta+\widehat{\beta}$ in (B.2).

(3) Let $K>1$. From Part (1), $u \in Y_{T}^{\alpha(\xi, \lambda)}$ for all $T \geq 0$. Moreover, we note that the result will follow, if we can show that $\|u\|_{\alpha(\xi, \lambda), T} \leq \rho(T)$, for some $0 \leq T \leq T_{0}$. Indeed, $\rho(T)$ is an increasing function of $T$, whereas $\|u\|_{\alpha, S} \geq\|u\|_{\alpha, T}$ for all $S \leq T$. 
We start by remarking that $\lim _{T \rightarrow+\infty} \frac{\|u\|_{\alpha(\xi, \lambda), T}}{\rho(T)} \leq \lim _{T \rightarrow+\infty} \frac{\|v\|_{\infty}}{\rho(T)}$ $=0$. As a consequence, either $\|u\|_{\alpha(\xi, \lambda), T} \leq \rho(T)$ for all $T \geq 0$, or there exists $\widetilde{T} \geq 0$ such that $\|u\|_{\alpha(\xi, \lambda), \widetilde{T}}=\rho(\widetilde{T})$. In the first case, the result is proven. In the second alternative, we can write

$$
\rho(\widetilde{T})=\|u\|_{\alpha(\xi, \lambda), \widetilde{T}}=\|\mathcal{H}(u)\|_{\alpha(\xi, \lambda), \widetilde{T}} \leq|\beta|\left|\phi_{3}\right| e^{-\left(\frac{1}{2}(1+\xi)-\alpha(\xi, \lambda)\right) \widetilde{T}}+\frac{\rho(\widetilde{T})}{2 K},
$$

using the estimate (B.8), and the fact that $u=\mathcal{H}(u)$, by Part (2). The definitions of $\rho$ and $T_{0}$ imply

$$
\frac{(2 K-1)}{2 K} \rho(\widetilde{T}) e^{-\alpha(\xi, \lambda) \widetilde{T}}=|\beta|\left|\phi_{3}\right| e^{-\frac{1}{2}(1+\xi) T_{0}} .
$$

Since we have assumed $\xi>-1$, the last two formulas give $\widetilde{T} \leq T_{0}$.

Proposition B.5. (bound on $\beta$ ) Let $m \neq-1$. For every $\delta>0, \Lambda \in \mathbb{R}$, there exists $\kappa_{1}=\kappa_{1}(\Lambda, \delta)>0$ such that for every $(\lambda, v, \xi) \in \mathcal{C}_{ \pm}$satisfying $0 \leq \lambda \leq \Lambda$ (resp. $\Lambda \leq \lambda \leq 0)$, and $\xi \geq-1+\delta$, there holds

$$
|\beta(\lambda, v, \xi)| \leq \kappa_{1}
$$

where $\beta(\lambda, v, \xi)$ is given in Proposition B.4(2).

Proof. If $\Lambda=0$, there is nothing to prove, therefore we take $\Lambda \neq 0$. We give the proof for $\Lambda>0$, the case $\Lambda<0$ being similar.

Suppose, by contradiction, that there exists a sequence $\left(\lambda^{(\nu)}, v^{(\nu)}\right.$, $\left.\xi^{(\nu)}\right)_{\nu \in \mathbb{N}}$ of $\mathcal{C}_{+}$, with $0 \leq \lambda^{(\nu)} \leq \Lambda, \xi^{(\nu)} \geq-1+\delta$, and for which $\lim _{\nu \rightarrow \infty}\left|\beta^{(\nu)}\right|=$ $\infty$, where $\beta^{(\nu)}:=\beta\left(\lambda^{(\nu)}, v^{(\nu)}, \xi^{(\nu)}\right), \nu \in \mathbb{N}$.

For each $\nu \in \mathbb{N}$, we set $u^{(\nu)}(t)=e^{-\alpha\left(\xi^{(\nu)}, \lambda^{(\nu)}\right) t} v^{(\nu)}(t)$, for all $t \geq 0$. By Remark 3.3, $u^{(\nu)}+1+\xi$ solves (3.6) and (3.7). Therefore Lemma 3.5, together with the choice of $U$ given in (3.20), implies that there is some $\kappa_{0}=\kappa_{0}(\Lambda)>0$ such that $\left|\xi^{(\nu)}\right|+\left\|u^{(\nu)}\right\|_{\infty} \leq \kappa_{0}$. Hence, up to a subsequence, we may assume that there exist $\lambda^{*} \in[0, \Lambda], \xi^{*} \in \bar{U} \cap[-1+\delta,+\infty)$, and $\zeta^{*} \in \mathbb{R}$ such that

$$
\lim _{\nu \rightarrow \infty} u^{(\nu)}(0)=\lim _{\nu \rightarrow \infty}\left(-\xi^{(\nu)}, \lambda^{(\nu)}, u_{3}^{(\nu)}(0)\right)=\left(-\xi^{*}, \lambda^{*}, \zeta^{*}\right) .
$$

Now we consider the solution $u^{*}$ of the initial-value problem $u^{\prime}+A\left(\xi^{*}\right) u+$ $N(u)=0, u(0)=\left(-\xi^{*}, \lambda^{*}, \zeta^{*}\right)$, where $A$ and $N$ are given by (3.12) and (3.13). Since, for every $\nu \in \mathbb{N}, u^{(\nu)}$ is uniformly bounded over $[0, \infty)$ by $\kappa_{0}$, it follows that the solution $u^{*}$ is defined for all $t \geq 0$. Let us show that $\lim _{t \rightarrow \infty} u_{2}^{*}(t)=0$. First note that $\left(u_{2}^{*}\right)^{\prime}(t)=u_{3}^{*}(t)=\lim _{\nu \rightarrow \infty} u_{3}^{(\nu)}(t)$ for all $t \geq 0$. Moreover, by Proposition 3.7(2) there exists a $t_{0} \geq 0$ such that for all $\nu \in \mathbb{N}, u_{3}^{(\nu)}(t)<0$ for every $t>t_{0}$. Thus, $u_{2}^{*}$ decreases over $\left(t_{0}, \infty\right)$, and $u_{2}^{*}(t)=\lim _{\nu \rightarrow \infty} u_{2}^{(\nu)}(t) \geq 0$ for all $t \geq 0$. Since $0 \leq \int_{t_{0}}^{+\infty} u_{2}^{(\nu)}(s) d s=-u_{1}^{(\nu)}\left(t_{0}\right) \leq \kappa_{0}$, for all $\nu \in \mathbb{N}$, Fatou's Lemma implies that $u_{2}^{*} \in L^{1}\left(t_{0}, \infty\right)$, and consequently that $\lim _{t \rightarrow \infty} u_{2}^{*}(t)=0$.

Let $K>1$ and consider $\nu \in \mathbb{N}$. Let $T_{0}^{(\nu)}$, be the value of $T_{0}$ given in Proposition B.3 and associated to $\left(\lambda^{(\nu)}, v^{(\nu)}, \xi^{(\nu)}\right)$ via Proposition B.4. Then 
$u^{(\nu)}(t)=\hat{u}(t)=\lim _{k \rightarrow \infty} \mathcal{H}^{k}(0)(t)$, for all $t \geq T_{0}^{(\nu)}$. Using (B.5) and (B.6), we find that

$$
\begin{aligned}
\left|u^{(\nu)}(t)-\mathcal{H}(0)(t)\right| & \leq \sum_{k=1}^{\infty}\left|\mathcal{H}^{k+1}(0)(t)-\mathcal{H}^{k}(0)(t)\right| \\
& \leq \frac{e^{-\alpha\left(2 t-T_{0}^{(\nu)}\right)}}{K-1}\|\mathcal{H}(0)\|_{\alpha, T_{0}^{(\nu)}}
\end{aligned}
$$

for all $t \geq T_{0}^{(\nu)}$, where $\alpha=\alpha\left(\xi^{(\nu)}, \lambda^{(\nu)}\right)$. Evaluating $\mathcal{H}(0)$, we obtain

$$
\left|u^{(\nu)}(t)-\beta^{(\nu)} e^{-\frac{1}{2}\left(1+\xi^{(\nu)}\right) t} \phi_{3}\right| \leq e^{-2 \alpha t} \frac{\left|\beta^{(\nu)}\right|\left|\phi_{3}\right|}{K-1},
$$

for all $t \geq T_{0}^{(\nu)}$, where we have used the fact that $\alpha>\frac{1}{4}\left(1+\xi^{(\nu)}\right)$. Therefore, we deduce that

$$
\left|u_{2}^{(\nu)}(t)+\frac{1}{2}\left(1+\xi^{(\nu)}\right) \beta^{(\nu)} e^{-\frac{1}{2}\left(1+\xi^{(\nu)}\right) t}\right| \leq e^{-2 \alpha t} \frac{\left|\beta^{(\nu)}\right|\left|\phi_{3}\right|}{K-1},
$$

for all $t \geq T_{0}^{(\nu)}$. But then

$$
\begin{aligned}
\left|u_{2}^{(\nu)}(t)\right| \geq & \left|\frac{1}{2}\left(1+\xi^{(\nu)}\right) \beta^{(\nu)} e^{-\frac{1}{2}\left(1+\xi^{(\nu)}\right) t}\right|-\mid u_{2}^{(\nu)}(t) \\
& +\frac{1}{2}\left(1+\xi^{(\nu)}\right) \beta^{(\nu)} e^{-\frac{1}{2}\left(1+\xi^{(\nu)}\right) t} \mid \\
\geq & \left|\beta^{(\nu)}\right| e^{-\frac{1}{2}\left(1+\xi^{(\nu)}\right) t}\left(\frac{1}{2}\left(1+\xi^{(\nu)}\right)-\frac{\left|\phi_{3}\right|}{K-1}\right),
\end{aligned}
$$

for all $t \geq T_{0}^{(\nu)}$, using again the relation $\alpha>\frac{1}{4}\left(1+\xi^{(\nu)}\right)$.

Now we let $K=1+\frac{4+\left(1+\kappa_{0}\right)^{2}}{\delta}$, and observe that $\delta<\left(1+\xi^{(\nu)}\right)<1+\kappa_{0}$. As a consequence, $K \geq 1+\frac{4\left|\phi_{3}\right|}{\left(1+\xi^{(\nu)}\right)}$, and from the definition of $T_{0}^{(\nu)}$, we obtain

$$
\begin{aligned}
\left|\beta^{(\nu)}\right| & =\frac{(2 K-1)\left(1+\xi^{(\nu)}\right)^{2}\left(4 \alpha-\left(1+\xi^{(\nu)}\right)\right)}{24 K^{2}\left|\phi_{3}\right|^{2}\left(1+\left|\frac{2 m}{m+1}\right|\right)} e^{\frac{1}{2}\left(1+\xi^{(\nu)}\right) T_{0}^{(\nu)}} \\
& \leq \frac{4}{\left(1+\xi^{(\nu)}\right)} e^{\frac{1}{2}\left(1+\xi^{(\nu)}\right) t}\left|u_{2}^{(\nu)}(t)\right|,
\end{aligned}
$$

for all $t \geq T_{0}^{(\nu)}$. Setting $t=T_{0}^{(\nu)}$ and using the bounds on $\xi^{(\nu)}$ and $\lambda^{(\nu)}$, we conclude that there exists a constant $C=C(\Lambda, \delta)>0$ such that

$$
\left|u_{2}^{(\nu)}\left(T_{0}^{(\nu)}\right)\right| \geq C, \quad \text { for all } \nu \in \mathbb{N} .
$$

On the other hand, recall that for every $t \geq 0, u^{(\nu)}(t) \rightarrow u^{*}(t)$ as $\nu \rightarrow \infty$, and that $\lim _{t \rightarrow \infty} u_{2}^{*}(t)=0$. Therefore, for any $\varepsilon>0$, there exists $S_{\varepsilon} \geq 0$ such that $\left|u_{2}^{*}(t)\right|<\varepsilon / 2$, for all $t \geq S_{\varepsilon}$. Moreover, there exists $N_{0} \in \mathbb{N}$ such that $\left|u_{2}^{(\nu)}\left(S_{\varepsilon}\right)-u_{2}^{*}\left(S_{\varepsilon}\right)\right|<\varepsilon / 2$, for all $\nu \geq N_{0}$. Hence, $\left|u_{2}^{(\nu)}\left(S_{\varepsilon}\right)\right|<\varepsilon$, and therefore $\left|u_{2}^{(\nu)}(t)\right|<\varepsilon$ for all $t \geq \max \left\{t_{0}, S_{\varepsilon}\right\}$, since $\left|u_{2}^{(\nu)}\right|$ decreases over $\left(t_{0}, \infty\right)$. From (B.9), we deduce that $T^{(\nu)} \leq \max \left\{t_{0}, S_{\varepsilon}\right\}$, for all $\nu \geq N_{0}$ and all $\varepsilon>C$, which contradicts our assumption that $\lim _{\nu \rightarrow \infty}\left|\beta^{(\nu)}\right|=\infty$ and therefore proves the desired result. 


\section{References}

[1] Amann, H.: Gewöhnliche Differentialgleichungen. de Gruyter Lehrbuch, vol. 2. Auflage, Stuttgart (1995)

[2] Brighi, B.: On a similarity boundary layer equation. Zeit. Anal. Anw. 21, 931-948 (2002)

[3] Brighi, B., Hoernel, J.-D.: Recent advances on similarity solutions arising during free convection. Progr. Nonlinear Differ. Equ. Appl. 63, 83-92 (2005)

[4] Brighi, B., Sari, T.: Blowing-up coordinates for a similarity boundary layer equation. Discrete Continuous Dyn. Syst. A 12, 929-948 (2005)

[5] Cheng, P., Minkowycz, W.J.: Free convection about a vertical flat plate embedded in a porous medium with application to heat transfer from a dike. J. Geophys. Res. 82, 2040-2044 (1977)

[6] Doedel, E.J.: AUTO, a program for the automatic bifurcation analysis of autonomous systems. Congr. Numer. 30, 265-384 (1981)

[7] Engelking, R.: General Topology. Heldermann, Berlin (1989)

[8] Evéquoz, G.: Global continuation for ODE systems over the half-line and study of Von Kármán's swirling flow problem. Thèse No. 3857, Ecole Polytechnique Fédérale de Lausanne (2007)

[9] Evéquoz, G.: Global continuation for first-order systems over the half-line involving parameters. Math. Nachr. 282, 1036-1078 (2009)

[10] Evéquoz, G.: Erratum to "Global continuation for first-order systems over the half-line involving parameters". Math. Nachr. 283, 630-631 (2010)

[11] Fitzpatrick, P.M., Pejsachowicz, J., Rabier, P.J.: The degree of proper $\mathrm{C}^{2}$ Fredholm mappings I. J. Reine Angew. Math. 427, 1-33 (1992)

[12] von Kármán, Th., Lin, C.C.: On the existence of an exact solution of the equations of Navier-Stokes. Comm. Pure Appl. Math. 14, 645-655 (1961)

[13] Liao, S.J.: A new branch of solutions of boundary-layer flows over a permeable stretching plate. Int. J. Nonlinear Mech. 42, 819-830 (2007)

[14] Magyari, E., Keller, B.: Backward free convection boundary layers in porous media. Transp. Porous Media 55, 285-300 (2004)

[15] McLeod, J.B.: The existence of axially symmetric flow above a rotating disk. Proc. R. Soc. Lond. A 324, 391-414 (1971)

[16] McShane, E.J.: Unified Integration. Academic Press, London (1983)

[17] Morris, J.: Nonlinear Ordinary and Partial Differential Equations on Unbounded Domains. Ph.D. Thesis, University of Pittsburgh (2005)

[18] Nield, D.A., Bejan, A.: Convection in Porous Media, 3rd edn. Springer, Berlin (2006) 
[19] Pejsachowicz, J., Rabier, P.J.: Degree theory for $\mathrm{C}^{1}$ Fredholm mappings of index 0. J. Anal. Math. 76, 289-319 (1998)

[20] Rabier, P.J.: Ascoli's Theorem for functions vanishing at infinity and selected applications. J. Math. Anal. Appl. 290, 171-189 (2004)

[21] Rabier, P.J., Stuart, C.A.: Boundary value problems for first order systems on the half-line. Top. Methods Nonlinear Anal. 25, 101-133 (2005)

[22] Rabier, P.J., Stuart, C.A.: A Sobolev space approach to boundary-value problems on the half-line. Commun. Contemp. Math. 7, 1-36 (2005)

G. Evéquoz

Institut für Analysis,

Universität Karlsruhe (TH),

Kaiserstraße 89,

76133 Karlsruhe,

Germany

e-mail: gilles.evequoz@math.uni-karlsruhe.de

Received: 16 January 2009.

Accepted: 02 March 2010. 